\title{
A CROSSLINKED COLLAGEN MEMBRANE VERSUS A NON- CROSSLINKED BILAYER COLLAGEN MEMBRANE FOR SUPPORTING OSTEOGENIC FUNCTIONS OF HUMAN BONE MARROW- MULTIPOTENT STROMAL CELLS
}

\author{
J.J. El-Jawhari ${ }^{1,2}$, K. Moisley ${ }^{1,3}$, E. Jones ${ }^{1}$ and P.V. Giannoudis ${ }^{1,4, *}$ \\ ${ }^{1}$ Leeds Institute of Rheumatic and Musculoskeletal Medicine, University of Leeds, Leeds, UK \\ ${ }^{2}$ Clinical Pathology Department, Mansoura University, Mansoura, Egypt \\ ${ }^{3}$ Institute of Medical and Biological Engineering, Faculty of Mechanical Engineering, \\ University of Leeds, Leeds, UK \\ ${ }^{4}$ Academic Unit of Trauma and Orthopaedic Surgery, Leeds Teaching Hospitals NHS Trust, Leeds, UK
}

\begin{abstract}
Barrier membranes are popularly used for guided bone regeneration (GBR). However, more knowledge is needed to assess how these membranes could be of therapeutic value when populated with native multipotent stromal cells (MSCs), particularly in the orthopaedic field. The present manuscript investigated the activities of human bone marrow-multipotent stromal cells (BM-MSCs) when loaded on two differently structured pure collagen membranes. A crosslinked collagen membrane (CS) was tested versus a non-crosslinked bilayer collagen membrane, Bio-Gide ${ }^{\circledast}(\mathrm{BG})$. Following loading with BM aspirate containing native MSCs, cell attachment to the membranes was examined using electron microscopy and flow cytometry. Furthermore, alkaline phosphatase (ALP) expression and calcium deposition levels were investigated for these BM-aspirateloaded membranes. Culture-expanded BM-MSCs were also used to load membranes and confirm the MSC functional data. All membranes supported BM-MSC attachment. However, larger numbers of attached BMMSCs were detected for CS as compared to BG $(p=0.0010)$. In osteogenic medium, ALP activity was higher for CS than BG $(p=0.0312)$. Total calcium deposition (not normalised to cell count) was also higher for CS than BG $(p=0.0073)$. Consistently, the normalised secreted vascular endothelial growth factor A (VEGF-A) levels were higher in BM-MSCs loaded on CS relative to BG $(p=0.0302)$. Collectively, both collagen membranes supported the osteogenic functions of BM-MSCs. However, CS was found to be overall superior, probably since it provided more BM-MSC attachment. These collagen membranes could potentially be used to improve GBR outcomes in orthopaedic applications.
\end{abstract}

Keywords: Bone, multipotent stromal cells, osteogenesis, repair, collagen membrane, guided bone regeneration.

*Address for correspondence: Peter V. Giannoudis MD, FACS, FRCS, Professor and Chairman, Academic Unit of Trauma and Orthopaedic Surgery/Honorary Orthopaedic and Trauma Consultant, Leeds General Infirmary, School of Medicine, University of Leeds, Leeds, UK.

Telephone number: +44 1133922750 Email: pgiannoudi@aol.com

Copyright policy: This article is distributed in accordance with Creative Commons Attribution Licence (http://creativecommons.org/licenses/by-sa/4.0/).

\section{Introduction}

Guided bone regeneration (GBR) approaches in orthopaedics usually involve staged surgeries for treating bone infections, large bone defects or fractures (Giannoudis et al., 2011; Masquelet and Begue, 2010). The Masquelet technique is an example of GBR surgery, where a cement spacer is used either as a prophylaxis or as a treatment for bone loss resulting from infection and tissue damage (Anagnostakos, 2017; Garg et al., 2011; Sivakumar et al., 2016). An induced membrane is a fibrous tissue resulting from the body reaction to the presence of polymethacrylate in the cement spacer. When the cement is removed during a second surgery, the empty space is packed with autograft material to help bone formation (Hammerle and Jung, 2003). The induced membrane has a vascular structure 
similar to that of the periosteum and is highly enriched in multipotent stromal cells (MSCs) as well as osteogenic and angiogenic inductive factors, such as bone morphogenic protein-2 (BMP-2) and vascular endothelial growth factor (VEGF) (Cuthbert et al., 2013; Giannoudis et al., 2011; Sivakumar et al., 2016). Despite the advantages of the induced membrane, this two-stage technique is usually time-consuming and costly (Arrington et al., 1996). Therefore, the use of a biocompatible membrane in a single surgical procedure with properties similar to that of naturallyformed membrane would pose a real advantage supporting endogenous MSC functions.

Barrier membranes have already been used in GBR surgeries, mainly for craniomaxillofacial regeneration, to enclose the repair site filled with bone autograft and promote faster bone healing (Dimitriou et al., 2012). As shown in animal studies and clinical applications in dental tissue regeneration, the composition and structure of these membranes could influence the efficacy of bone regeneration (Dimitriou et al., 2012). Bio-absorbable membranes made of natural materials, such as collagen and chitosan, show excellent biocompatibility but are different in their mechanical properties, with some having fast in vivo degradation rate. However, relatively rapid degradation of barrier membranes has been proposed as a cause of spontaneous periodontal healing (Elgali et al., 2017). On the other hand, the use of synthetic bio-absorbable membranes, such as aliphatic polyesters, has been associated with high healing rates of segmental mandibular defects (Elgali et al., 2017). However, such synthetic bio-absorbable membranes could cause moderate cytotoxic reactions and decrease cell adhesion (Alpar et al., 2000). Non-absorbable membranes, such as polytetrafluoroethylene, constitute another group of membranes that provide good mechanical support for cell attachment, with stable structural integrity. However, these membranes require a second surgery for removal, which increases the risk of infection (Lee and Kim, 2014; Marouf et al., 2000). More knowledge is needed to understand how these membranes and their structure support the bone-healing-related functions of human MSCs, particularly in the orthopaedic field.

Collagen is the most abundant extracellular matrix (ECM) component in many tissues, including bone, supporting cell survival and functions (Hennessy et al., 2009; Popov et al., 2011). El-Jawhari et al. (2015) demonstrated that collagen incorporation within hydroxyapatite scaffolds significantly enhances attachment and proliferation of MSCs when loaded with bone marrow (BM) aspirates. As well as being highly biocompatible and flexible, collagen-based materials are clinically suitable for supporting tissue regeneration (Chevallay and Herbage, 2000; Stoppel et al., 2015). Collagen membranes have been assessed using animal models and have been found to promote successfully MSC attachment, growth and osteogenic differentiation (Li et al., 2011; Lotfi et al., 2016; Yang et al., 2015). These advantages have provided the rationale for fabricating pure collagen membranes for regenerative applications in maxillofacial, dental and neuro-surgeries (Dimitriou et al., 2012; Schwarz et al., 2017). In contrast, using these collagen membranes loaded with BM aspirate containing MSCs is not yet popular in orthopaedic GBR treatments, although, the development of such novel one-stage intramusculartype procedures could be highly effective.

The purpose of the present study was to simulate the in vivo clinical situation and to understand further how native MSCs can attach to collagen membranes with different structures and support osteogenesis and angiogenesis. The membranes were loaded with $\mathrm{BM}$ aspirates to evaluate attachment, survival and functions of rare native bone marrow-MSCs (BM-MSCs), which were characterised according to their surface phenotype as CD45 ${ }^{\text {negative }} C D 271^{\text {high }}$ cells (Cuthbert et al., 2012; El-Jawhari et al., 2017). Additionally, culture-expanded BM-MSCs were used to confirm the biological characteristics of native MSCs loaded on these membranes. These included alkaline phosphatase (ALP) activity, mineral deposition and VEGF-A production/expression. The present study could help the understanding of how MSCs can promote osteogenesis when they colonise collagen membranes in vivo and, eventually, developing novel GBR approaches for large bone defects/fractures.

\section{Materials and Methods}

\section{BM samples and BM-MSC isolation in culture}

24 samples of BM aspirates were included in the study. The BM aspirates were collected from consented patients undergoing orthopaedic procedures in Leeds General Infirmary University Teaching Hospital. The work was carried out in accordance with the Code of Ethics of the World Medical Association (Declaration of Helsinki), National Research Ethics Committee Yorkshire and Humber-Leeds East (number 06/ Q1206/127). The donors included 15 males and 9 females with an age range of 18-63 years and a median of 40 years. From each donor, $10 \mathrm{~mL}$ of $\mathrm{BM}$ were aspirated from the anterior iliac crest of the pelvis, always by the same surgeon (P.V. Giannoudis), as previously described (El-Jawhari et al., 2015). The isolation of BM-MSCs in culture was performed as described before and using the erythrocyte lysis technique (El-Jawhari et al., 2015; Fragakis et al. 2018). In brief, BM aspirate samples were processed to remove erythrocytes using $0.86 \%$ ammonium chloride solution (Vickers Laboratories, Pudsey, UK) and, after wash with phosphate-buffered saline (PBS), BM cells were seeded at $2 \times 10^{6}$ cells per $2,500 \mathrm{~mm}^{2}$ flask and cultured. The StemMACS ${ }^{\mathrm{TM}}$ MSC Expansion Medium (Miltenyi Biotec, Bergisch Gladbach, Germany) was used to support MSC plastic-adhesion and proliferation. The culture medium was changed after $48 \mathrm{~h}$, then, half-changed 
twice a week until $60-80 \%$ confluency, when cells were detached using trypsin (Sigma-Aldrich) for further passage. The culture-expanded MSCs were used for the experiments after 3-4 passages.

\section{Collagen membranes: loading and culture}

The two membranes included in the study were $100 \%$ porcine collagen type I and III, ready-made by Geistlich Pharma, Wolhusen, Switzerland. A non-crosslinked collagen membrane, Bio-Gide ${ }^{\circledR}$ (BG; $1 \mathrm{~mm}$-thick) had one cell-non-permeable layer and another cell-permeable layer. The second membrane, a crosslinked collagen membrane (CS, $6 \mathrm{~mm}$ thickness) was cell-permeable from all sides. To standardise the total surface area of both membranes, the original membranes were cut vertically or transversely in a crosswise direction at right angles to the long axis, while keeping the original thickness for both membranes. A total surface area of $60 \mathrm{~mm}^{2}$ was set for each membrane as the standard for all comparisons. As recommended by the manufacturer, BG was soaked in the MSC expansion medium at $37^{\circ} \mathrm{C} 24 \mathrm{~h}$ before being loaded with cells.

The loading was performed under sterile conditions. For each donor sample, the loading was performed equally for both membranes simultaneously. According to the experiment type, either the same volume of unprocessed BM aspirate sample [i.e. $400 \mu \mathrm{L}$, contained on average 4271 CD45 ${ }^{\text {negative }}$ CD271 $1^{\text {high }}$ cells (range: $549-9646$ cells)] or the same number of culture-expanded MSCs (range: $2-3 \times 10^{5}$ MSCs suspended in $400 \mu \mathrm{L}$ medium per $\mathrm{cm}^{2}$ ) were used. Carried by forceps, each membrane was placed flat inside a well of a 24 -well plate. The loading was carefully applied drop by drop on the top of the cell permeable layer of either BG or CS surfaces. The membranes were left for 1,2 or $3 \mathrm{~h}$ at $37^{\circ} \mathrm{C}$ to allow for cell adherence, with gentle movement every $30 \mathrm{~min}$ to encourage further cell attachment. The membranes were gently washed with PBS before being moved into new 24-well plates, covered with medium and cultured for defined periods, according to the experiment. The StemMACS ${ }^{\mathrm{TM}}$ MSC Expansion medium or the osteogenic medium formed of low-glucose Dulbecco's Modified Eagle Medium (DMEM), $10 \%$ foetal calf serum (FCS), $100 \mathrm{nM}$ dexamethasone, $10 \mathrm{mM} \beta$-glycerophosphate and $0.05 \mathrm{mM}$ ascorbic acid (all from Sigma-Aldrich) were used according to the experiment plan.

\section{Electron and confocal microscopy}

Membranes were used unloaded or loaded (with BM aspirate or culture-expanded BM-MSCs), then, further cultured in MSC expansion or osteogenic medium according to the experiment plan. The membranes were fixed in $3.7 \%$ formaldehyde before electron and confocal microscopy analysis. Environmental scanning electron microscopy (ESEM) was used to examine the topography of the membrane surface and cell attachment following 2-week cultivation, using an S-3700N scanning electron microscope
(Hitachi, Berkshire, UK) as previously described (ElJawhari et al., 2015). Images of the hydrated samples were acquired under low vacuum at $-20^{\circ} \mathrm{C}$. Mineral deposition (calcium/phosphorus) on the membrane surfaces, under osteogenic conditions, was semiquantitatively assessed using energy-dispersive X-ray spectroscopy (EDS), as reported before (Kouroupis et al., 2013). EDS mapping images were acquired to a depth of $2 \mathrm{~mm}$, over an $8 \mathrm{~min}$ acquisition time and each analysis was performed in triplicate.

For confocal imaging, the fixed membranes were permeabilised in $0.1 \%$ Tween 20 for 15 min to allow intracellular staining. Then, the membranes were stained for $1 \mathrm{~h}$ in the dark with $0.1 \% 4^{\prime}, 6$-diamidino2-phenylindole (DAPI) with or without phalloidin $(1 \mu \mathrm{g} / \mathrm{mL}$ in PBS), which was used for actin structure staining (Sigma-Aldrich), and washed in PBS before imaging. The membranes were imaged using the SP8 confocal laser scanning microscope (Leica) using $405 \mathrm{~nm}$ and $540 \mathrm{~nm}$ excitation wavelength. Images were captured using a $40 \times / 0.80 \mathrm{~W}$ U-V-I NA water dipping objective (Leica).

\section{Flow cytometry}

An indirect approach using flow cytometry was applied for evaluating the BM-MSC attachment to the collagen membranes after 1, 2 or $3 \mathrm{~h}$ of loading with BM aspirates. Samples from the pre- and post-loading BM fractions (5 different BM aspirates) were stained with a haematopoietic lineage marker, CD45 (563879, BD Biosciences), and a native BM-MSC marker, CD271 (130-112-601, Miltenyi Biotec). Native BMMSCs were quantified as CD $45^{\text {negative }}$ CD271 ${ }^{\text {high }}$ cells, as reported before (Cuthbert et al., 2012; El-Jawhari et al., 2017). The CountBright ${ }^{\mathrm{TM}}$ Absolute Counting Beads (Life Technologies) were used for absolute counting of these cells, according to the manufacturer's instructions. The number of CD $45^{\text {negative }} \mathrm{CD} 271^{\text {high }}$ cells attached to the membranes were calculated by subtraction of the post-loading cell number from the pre-loading cell number, considering pre-loading and post-loading BM volumes.

Flow cytometry was also used for quantifying the survived BM-MSCs within the membranes after 1, 2 or 3 weeks of culture in the expansion medium. Five different samples were analysed for direct counting of cells retrieved following membrane digestion. Membranes were digested using $0.25 \%$ collagenase type I (07902, Stem Cell Technologies Ltd) to release cells, as described previously (El-Jawhari et al., 2015). The cell surface levels of CD271 are reduced in culture (Battula et al., 2009), thus, the digested cell fractions were stained for the known positive markers of culture-expanded BM-MSCs (Dominici et al., 2006), CD90 (MCA90F, BIO-RAD) and CD73 (130-095-182, Miltenyi Biotec), as well as the negative marker CD45. Live cells were identified using the live and dead cell dyes, calcein violet and aqua, respectively (Life Technologies). Absolute counts of live BMMSCs were calculated using the CountBright ${ }^{\mathrm{TM}}$ Absolute Counting Beads (Life Technologies). All 
flow cytometry data were acquired on LSR-II flow cytometer and analysed using DIVA software (BD Bioscience).

\section{BM-MSC osteogenesis assessment}

Alizarin red Sstaining was performed to detect calcium deposits. BG and CS were loaded with BM aspirates and cultured for 3 weeks in osteogenic medium. Membranes were fixed in $3.7 \%$ formaldehyde (Sigma-Aldrich), paraffin-wax-embedded and sectioned. Alizarin red S (Sigma-Aldrich) aqueous solution was prepared as $40 \mathrm{mM}$ in distilled water at $\mathrm{pH}$ 4.1. The slides were stained for $10 \mathrm{~min}$ at room temperature, then rinsed 3 times in distilled water. The slides were scanned using the Aperio Digital Pathology Scanner (Leica) and viewed on ImageScope software (Leica).

ALP activity displayed by culture-expanded BMMSCs (from six different donors) that were loaded on the membranes was measured following 2-week culture in osteogenic or control StemMACS ${ }^{\mathrm{TM}}$ MSC Expansion medium, as described before (Kouroupis et al., 2013). Membranes were lysed in $0.1 \times$ Triton- $X$ solution (micelle concentration: $0.2 \mathrm{mM}$ at $20^{\circ} \mathrm{C}$ ) and exposed to three cycles of freezing-thawing to release the cellular contents. ALP activity was measured using the Alkaline Phosphatase Activity colorimetric kit (BioVision) according to the manufacturer's instructions. The measurements of ALP activity were normalised to DNA levels, which were calculated using the Quant-iT PicoGreen dsDNA Assay Kit (Life Technologies), as per the manufacturer's instructions.

Calcium assays were performed after 3 weeks of culture in the osteogenic or control expansion medium. Membranes loaded with culture-expanded BM-MSCs from five different donors were treated for $16 \mathrm{~h}$ at $4{ }^{\circ} \mathrm{C}$ with $0.5 \mathrm{M} \mathrm{HCl}$ solution and mild agitation to extract calcium. Calcium levels were analysed using a colorimetric kit (Calcium Liquid, Sentinel Diagnostics, Milan, Italy) as recommended by the manufacturer. The optical density readings for calcium and ALP assays were acquired using a Multiscan EX reader and analysed using Ascent ${ }^{\mathrm{TM}}$ software (ThermoFisher Scientific).

\section{Enzyme-linked immunosorbent assay (ELISA) for VEGF-A}

Secreted levels of VEGF-A were quantified in the culture supernatants of BM-MSC-loaded membranes (culture-expanded BM-MSCs from five different donors) after 1 and 3-week culture in either osteogenic or expansion medium. Culture supernatants were collected at these time points and kept frozen until analysis. VEGF-A colorimetric ELISA kit (KHG0111, Life Technologies) was used according to the manufacturer's instructions. The optical density was measured using Multiscan EX reader and analysed using Ascent ${ }^{\mathrm{TM}}$ software (ThermoFisher Scientific). The effect of medium change dilution on VEGF-A concentration was considered during data analysis.
Quantitative PCR for lineage markers, ALP and VEGF

Both membranes were loaded with either fresh BM aspirates (from three different donors) or cultureexpanded BM-MSCs (three different cultures) for $3 \mathrm{~h}$. Then, the loaded membranes were either cultured in expansion or osteogenic medium for 1 or 3 weeks. At each time point, membranes were washed in PBS. RNA was extracted directly from the membranes using the Single Cell RNA purification Kit (P4-0164, Geneflow Ltd, Lichfield, UK) by using a vortex to ensure cell lysis. The complementary DNA (cDNA) was produced from $200 \mu \mathrm{g}$ of RNA using the Reverse Transcription Master Mix (FLUIDIGM UK Ltd, Cambridge, UK). TaqMan probes (ThermoFisher Scientific) for VEGF-A (HS00900058_m1), VEGF-C (HS01099206_m1), CD45 [protein tyrosine phosphatase receptor type c (PTPRC), Hs00894734_ m1], CD73 (NT5E, Hs00159686_m1), CD90 (Thy1, Hs00174816_m1) and ALPL (Hs01029144_m1) were used for the assays. Real-time PCR assays were run on QuantStudio ${ }^{\text {TM }} 7$ Flex Real-Time PCR System, 384well (ThermoFisher Scientific). The gene expression levels were calculated relative to the housekeeping control gene, hypoxanthine guanine phosphoribosyl transferase (HPRT1, TaqMan probe, Hs02800695_m1) as previously published (Churchman et al., 2012). Negative controls of no RNA and no probe were included in each PCR run.

\section{Statistics}

Statistical analysis was performed using Prism software (version 7.0a; GraphPad). Comparative statistical tests (specified in the figure legends) were applied according to the normal distribution of the data tested using the Shapiro-Wilk normality test. All comparisons were paired as the same donor samples were used for both membranes. The difference between the groups was considered statistically significant for $p<0.05$.

\section{Results}

Visualisation of BM cell attachment to the collagen membranes

BG and CS membranes were loaded with the same volume of BM aspirate sample and cultured for 2 weeks. Then, ESEM was used to image the membrane surfaces. When compared with the unloaded BG, flattened structures were stretched over the surface of the cell-permeable layer of loaded BG. This observation suggested the presence of cells attached to the membrane (Fig. 1, left). The surfaces of the non-permeable layer of unloaded and loaded BG were also examined and the collagen fibres were tightly arranged as compared to the permeable surface, preventing any cell attachment or ingrowth (Fig. 1, middle). Additionally, the pores of the loaded CS were partially or fully filled as compared to the 
unloaded membrane (Fig. 1, right). Altogether, data suggested that both collagen membranes allowed BM cell attachment.

$\mathrm{BM}$ aspirates are composed of a heterogeneous mixture of cells with very small numbers of MSCs (El-Jawhari et al., 2015). Thus, the specific BM-MSC attachment to the membranes was evaluated using confocal microscopy after loading both membranes with the same numbers of culture-expanded BMMSCs. The loaded membranes were cultured for 2 weeks before confocal imaging. DAPI staining for nuclei was used to indicate the presence of BM-MSCs attached to collagen membranes. The unloaded BG and CS showed only background reflection of the membranes but no DAPI staining (Fig. 2a, top panels, left and right, respectively). In contrast, both MSCloaded BG and CS showed similarly positive staining with DAPI (Fig. 2a, lower panels, left and right, respectively). In addition to DAPI, phalloidin staining was detected, showing the actin structure of BMMSCs attached to both BG and CS (Fig. 2b, left and right, respectively). Collectively, confocal imaging helped to visualise and confirm the attachment of BM-MSCs to BG and CS membranes.

Quantification of BM-MSC attachment to the collagen membranes

Attachment of native BM-MSCs to the membranes was quantified by flow cytometry. Both membranes were loaded with the same BM aspirate for 1, 2 or $3 \mathrm{~h}$. Then, the number of attached BM-MSCs was calculated by subtraction of post-loading from preloading BM-MSC numbers. For both pre-loading and post-loading BM aspirates, the native BM-MSCs were identified as being CD45 $5^{\text {negative }} \mathrm{CD} 271^{\text {high }}$ and counted by flow cytometry using counting beads (Cuthbert et al., 2012; El-Jawhari et al., 2017). Similarly, other attached BM cells were counted and identified based on their size and CD45 expression (El-Jawhari et al., 2015) as CD45 ${ }^{\text {negative }}$ (containing MSCs as well as erythroid and endothelial cells), CD45 positive SSC (Side Scatter) ${ }^{\mathrm{med} / \text { high }}$ (myeloid-monocyte lineage cells) and CD45 ${ }^{\text {positive }}$ SSC ${ }^{\text {low }}$ (lymphocyte lineage cells). An example of this gating strategy is illustrated in Fig. 3a.

For BM aspirates from five different donors, the mean number of CD45 $5^{\text {negative }} \mathrm{CD} 271^{\text {high }}$ cells in $400 \mu \mathrm{L}$ of full BM aspirate (pre-loading samples) was 4271 cells (range: 549-9646 cells). The mean number of CD45 ${ }^{\text {negative }} \mathrm{CD} 271^{\text {high }}$ cells in the postloading samples after $3 \mathrm{~h}$ of loading was 1767 cells for BG and 734 for CS (Fig. 3b, upper panel), indicating a successful attachment of BM-MSCs to both membranes. However, the trend of lower CD45 ${ }^{\text {negative }}$ CD271 $1^{\text {high }}$ cell numbers in post-loading samples for CS relative to BG suggested better MSC attachment for CS. The mean number of myeloidmonocyte lineage cells in the pre-loading samples was $7.3 \times 10^{6}$ cells. The myeloid-monocyte lineage cells in the post-loading samples were counted as $3.8 \times 10^{6}$ and $1 \times 10^{6}$ cells after $3 \mathrm{~h}$ of loading with BG and CS, respectively (Fig. 3b, upper panel). As well, a mean of $1.6 \times 10^{6}$ lymphocyte-lineage cells was counted in pre-loading samples and $0.9 \times 10^{6}$ and $0.4 \times 10^{6}$ cells in post-loading samples following $3 \mathrm{~h}$ of loading with BG and CS, respectively (Fig. 3b, upper panel). Data showed a trend of lower numbers

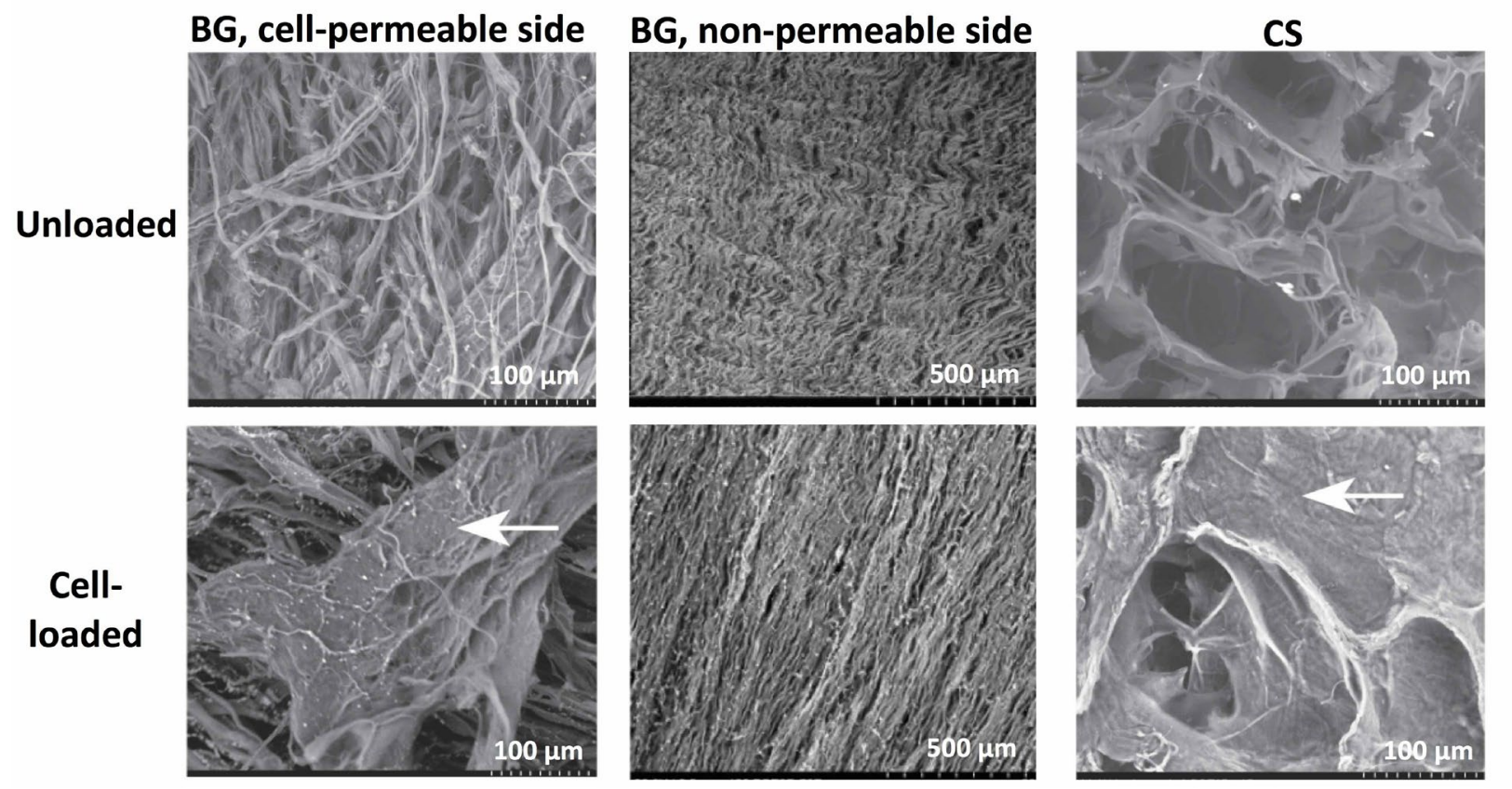

Fig. 1. ESEM imaging of collagen membranes. ESEM imaging of unloaded (upper panels) and loaded (lower panels) membranes: surface of the cell-permeable layer of BG (left), cell-non-permeable layer of BG (middle) and CS (right). Membranes were loaded with $400 \mu \mathrm{L}$ of BM aspirates for $3 \mathrm{~h}$ and cultured in expansion medium for 2 weeks and, finally, formalin-wax-fixed for imaging. Hydrated samples were acquired under low vacuum at $-20{ }^{\circ} \mathrm{C}$. White arrows indicate sites of the possible cell attachment to the loaded membranes. 

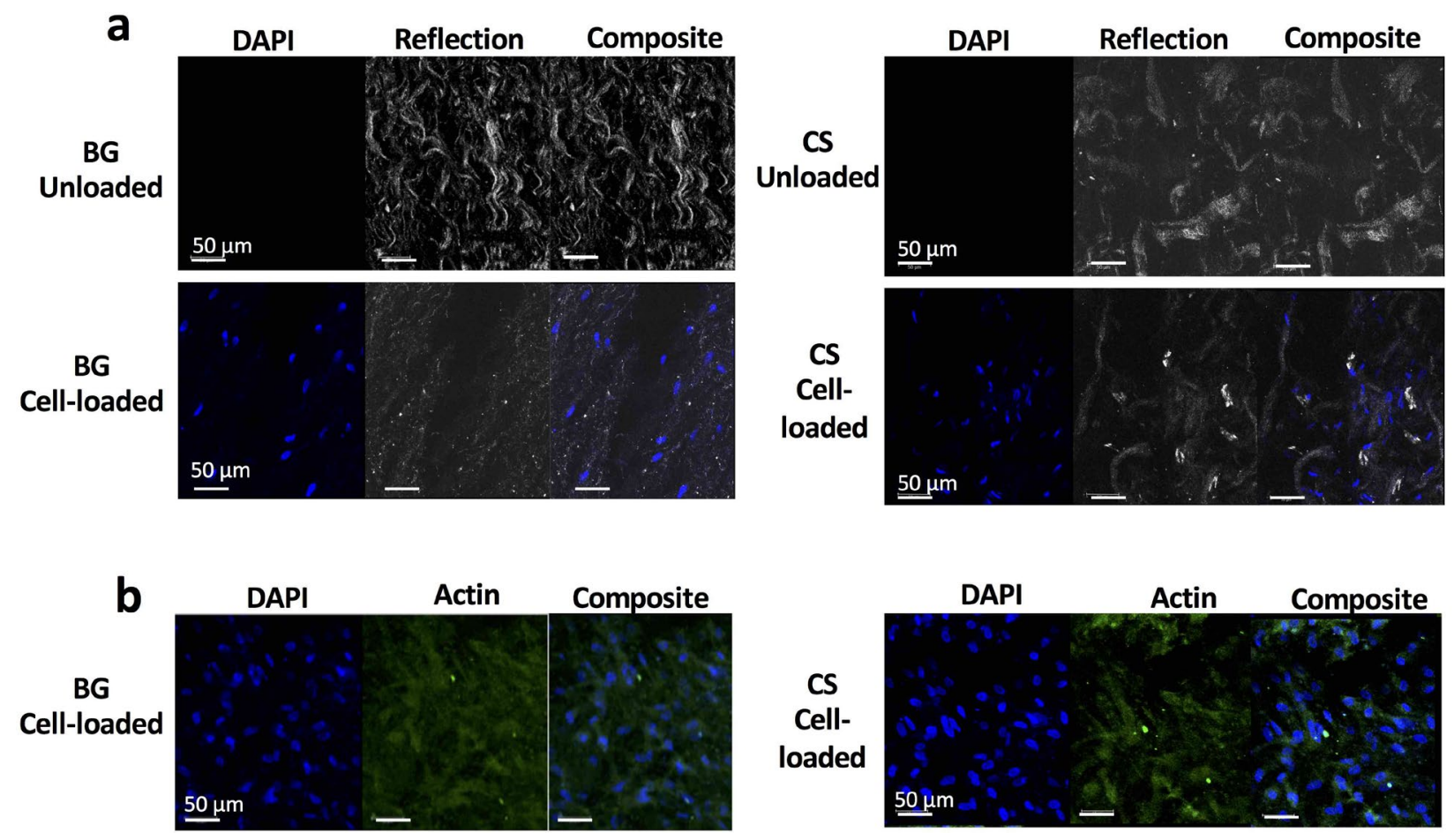

Fig. 2. Confocal imaging of collagen membranes. (a) Confocal images for BG (left) and CS (right) without loading (upper panels) or after loading with culture-expanded BM-MSCs (lower panels). Membranes were equally loaded for $3 \mathrm{~h}$ with 2-3 $\times 10^{5}$ culture-expanded BM-MSCs per $\mathrm{cm}^{2}$ and cultured in the expansion medium for 2 weeks before fixation and staining with DAPI. Images for DAPI staining, reflective light or composite are shown for each condition. (b) Confocal images for BG (left) and CS (right) after loading with culture-expanded BM-MSCs. The membranes were equally loaded for $3 \mathrm{~h}$ with 2-3 $\times 10^{5}$ culture-expanded BM-MSCs per $\mathrm{cm}^{2}$ and cultured in expansion medium for 2 weeks before fixation and staining with DAPI and actin. Images for DAPI (blue), actin (green) and composite staining are shown for each membrane.

of myeloid-monocyte and lymphocyte lineage cells in post-loading samples after loading CS as compared to BG, implying a better attachment of these cells to CS. Together, these findings demonstrated the successful attachment of different BM cells to the collagen membranes within a few hours of loading with BM aspirates and indicated some differences between BG and CS.

The median percentage of $\mathrm{CD} 45^{\text {negative }} \mathrm{CD} 271^{\text {high }}$ cells among other BM cells was $0.03 \%$ in pre-loading samples and 0.026 and $0.05 \%$ in post-loading samples for BG and CS, respectively (Fig. 3b, lower panel). Furthermore, the median percentage of myeloidmonocyte lineage cells was $53 \%$ and $57.3 \%$, in the pre-and post-loading sample, respectively (Fig. 3b, lower panel). For lymphocyte lineage cells, $15.7 \%$ and $16.8 \%$ were detected as the median percentages in pre- and post-loading samples, respectively (Fig. $3 \mathbf{b}$, lower panel). No statistically significant difference was detected for these data before and after loading. The comparable percentages of MSCs and other BM cells before and after loading of the membranes suggested that no BM cells, within early hours of incubation, preferentially attached to the membranes.

The number of attached BM-MSCs to CS was similar after 1,2 or $3 \mathrm{~h}$ of incubation but, for BG, $2 \mathrm{~h}$ were needed (in one sample) for a larger MSC attachment (Fig. 3c). Donor-related variations in attached CD45 ${ }^{\text {negative }} \mathrm{CD} 271^{\text {high }}$ cell numbers were observed, as reported before (Cuthbert et al., 2012; El-Jawhari et al., 2017). Collectively, for 1, 2, and $3 \mathrm{~h}$ of incubation, paired comparisons showed larger numbers of CD45 $5^{\text {negative }} \mathrm{CD} 271^{\text {high }}$ cells attached to CS than BG (2.5-fold difference, $p=0.0010$ ) (Fig. 3d). These data confirmed that CS was better for aiding the attachment of BM-MSCs than BG.

Quantification of live BM-MSCs retained on the collagen membranes in long-term culture

To assess how CS and BG supported long-term MSC survival, membranes loaded for $3 \mathrm{~h}$ with $\mathrm{BM}$ aspirates were cultured in expansion medium for 1, 2 or 3 weeks before membrane digestion with collagenase. Cells retrieved after digestion were analysed by flow cytometry and live BM-MSCs were characterised as aqua $^{-}$calcein-violet ${ }^{+}$CD $45^{-} \mathrm{CD}^{+} \mathrm{CD}^{+} 3^{+}$cells and counted using counting beads (El-Jawhari et al., 2015). The live BM-MSC counts of three different BM samples, retrieved from digested membranes were compared after 1, 2 and 3 weeks of culture. Data demonstrated a steady increase over time indicative of MSC proliferation on both membranes; however, with a trend of larger MSC numbers for CS than BG (Fig. 4a). Analysis of the numbers of retrieved MSCs 
a

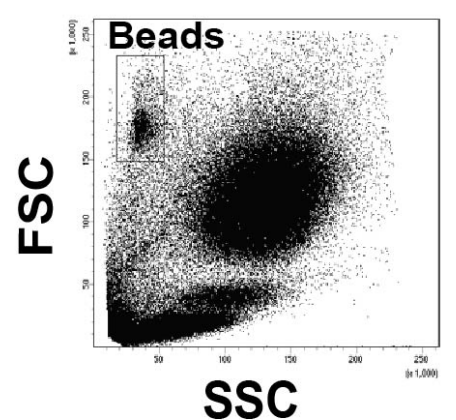

SSC

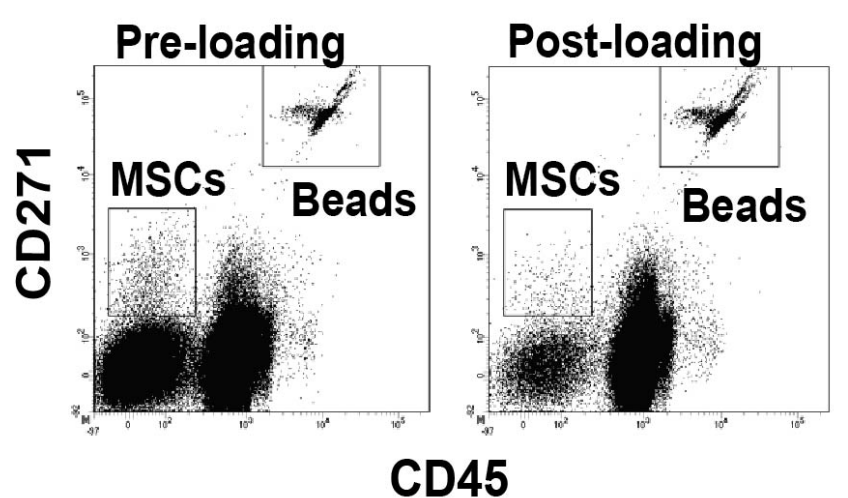

CD45

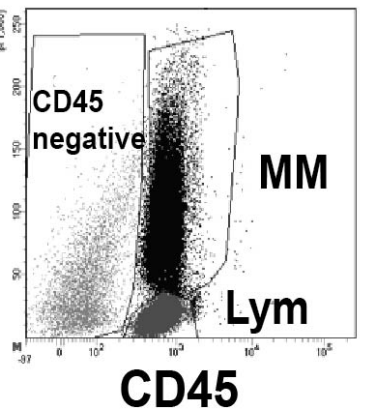

b

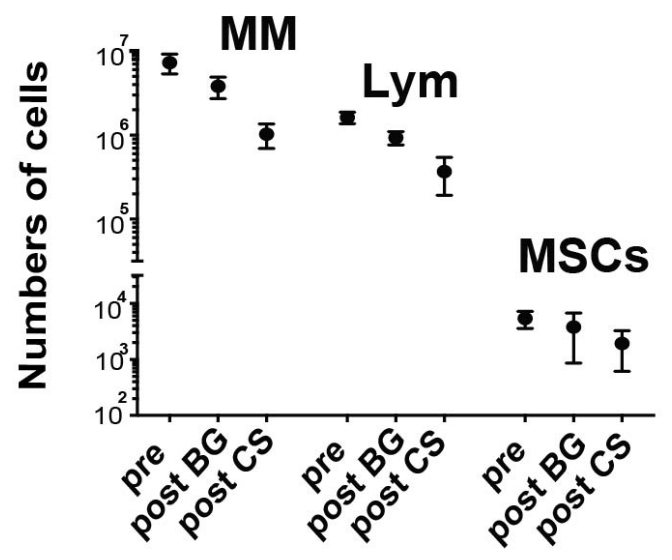

C
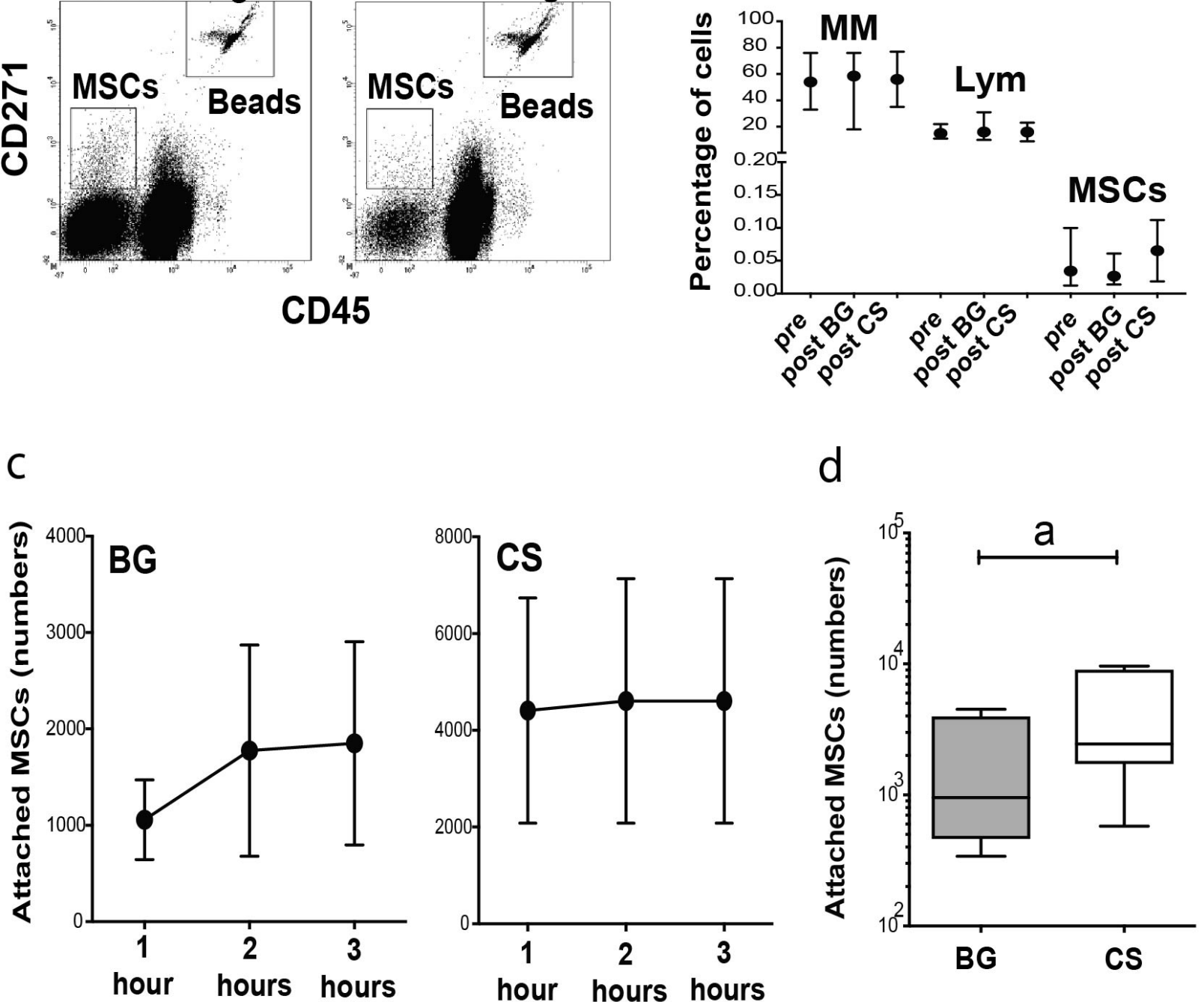

Fig. 3. Attachment of BM aspirate cells loaded on collagen membranes. (a) Using flow cytometry, BMMSCs were identified as being CD45 ${ }^{\text {negative }} \mathrm{CD} 271^{\text {high }}$ and other BM cells as CD45 $5^{\text {negative }}, \mathrm{CD} 45^{\text {positive }} \mathrm{SSC} \mathrm{C}^{\text {med/high }}$ (myeloid-monocyte-lineage cells) and CD45 positive SSC low (lymphocyte lineage cells). (b) Numbers (upper panel) and percentages (lower panel) of native BM-MSCs, lymphocyte (Lym) and myeloid-monocyte lineage cells (MM) in pre-loading and post-loading BM samples (mean of 5 different samples with standard error). (c) Numbers of attached native BM-MSCs over three loading times (1, 2 and $3 \mathrm{~h})$ for BG and CS (mean of 3 different samples with standard error represented). (d) Overall comparison of native BMMSC numbers attached to the membranes (median of 5 samples with an interquartile range represented; a $p<0.05$; Wilcoxon matched-pairs signed rank test). 
(samples from five different donors) after 3 weeks in culture showed that significantly more live BM-MSCs were released from CS than from BG (mean of 2.5fold, $p=0.0039$ ) (Fig. 4b).

If haematopoietic-lineage cells were similarly retained on the collagen membranes following several weeks of culture was assessed. Interestingly, a trend of decreasing numbers of myeloid-monocytelineage cells was noted when comparing cell numbers over 1, 2 and 3 weeks for both membranes (Fig. 4c, upper panel). A similar pattern of reduced numbers over time was detected for lymphocyte lineage cells (Fig. 4c, lower panel). For both cell types, more retained cells were found for CS than BG. Together, these results indicated that while MSC numbers were increasing with long-term culture on BG and CS, the numbers of haematopoietic-lineage cells decreased on these membranes over time.

To further confirm the changes in the populations retained on the membranes, as detected by flow cytometry, qPCR was performed to measure gene expression levels of MSC (CD73 and CD90) and haematopoietic-lineage cell (CD45) markers over the long culture period. BM aspirates from three different donors were loaded on the membranes for $3 \mathrm{~h}$ and, then, cultured for 1 or 3 weeks. The transcripts levels of PTPRC (CD45) were significantly lower after 3-week culture as compared to 1-week culture for both BG and CS ( $p=0.0312)$ (Fig. 4d), indicating decreased numbers of haematopoieticlineage cells over time in culture. In contrast, the transcript levels of Thy1 (CD90) and 5'-nucleotidase ecto [NT5E (CD73)] were significantly higher when measured after 3-week culture as compared to 1-week culture and for both BG and CS $(p=0.0312$, both markers for CS; $p=0.0328$ and $p=0.0312$, CD90 and CD73, respectively, for BG) (Fig. 4d), indicating increased numbers of MSCs over time in culture. In summary, both collagen membranes supported survival and proliferation of BM-MSCs rather than
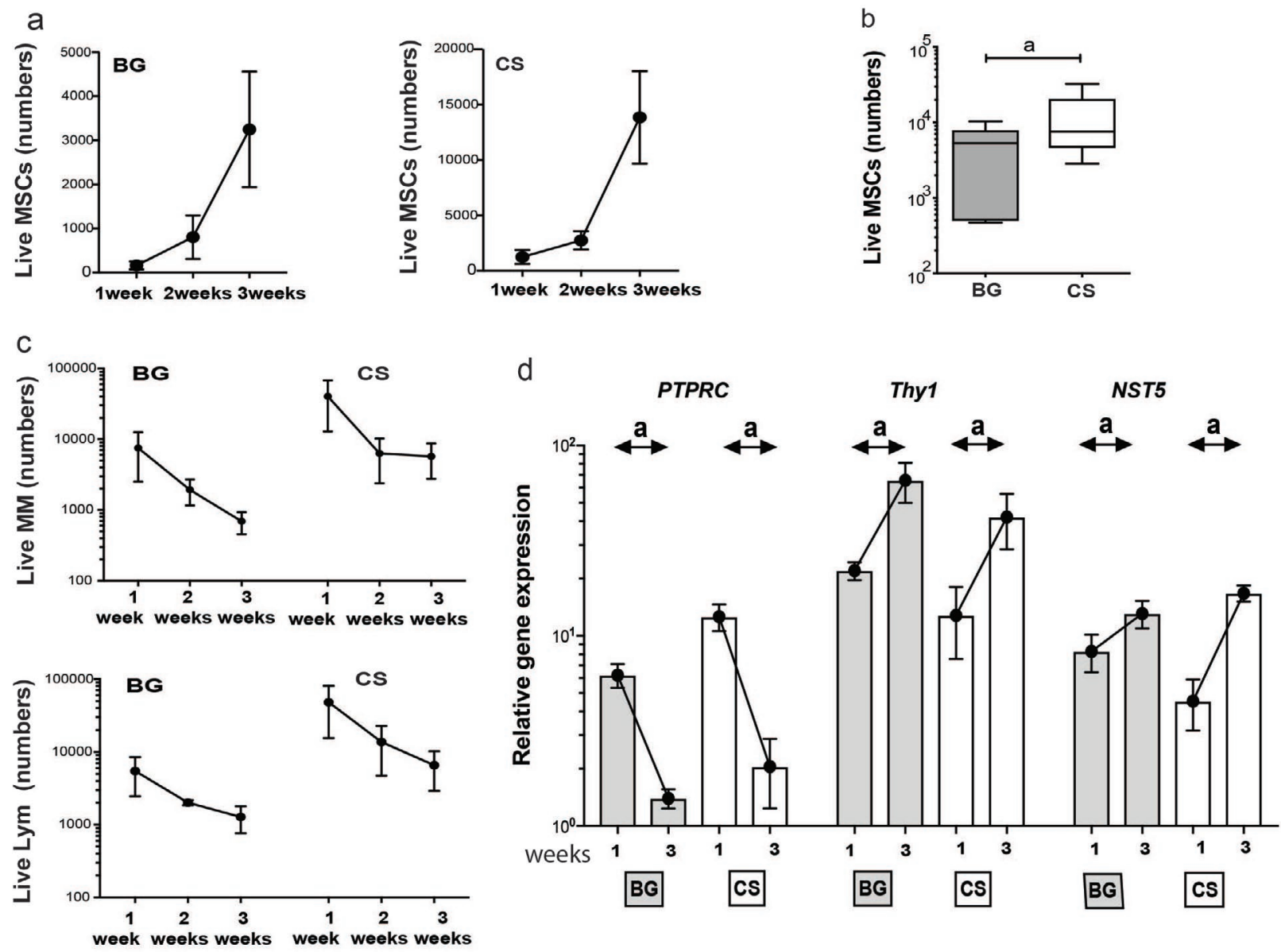

Fig. 4. Survival of BM cells retained on collagen membranes after culture. (a) Numbers of live BM-MSCs (quantified as aqua ${ }^{-}$calcein-violet ${ }^{+} \mathrm{CD} 45^{-} \mathrm{CD} 90^{+} \mathrm{CD}^{+} 3^{+}$cells) retrieved after the cultured membranes were digested (mean of 3 different samples with standard error represented). (b) Comparison of live BM-MSC numbers (quantified as aqua ${ }^{-}$calcein-violet ${ }^{+} \mathrm{CD} 45^{-} \mathrm{CD} 90^{+} \mathrm{CD} 3^{+}$cells) retrieved after cultured membranes were digested (median of 5 different samples with an interquartile range represented; ${ }^{a} p<0.05$; Wilcoxon matched-pairs signed rank test). (c) Numbers of live BM haemopoietic-lineage cells retrieved after the cultured membranes were digested (mean of 3 different samples with the standard error represented). (d) Gene expression levels of PTPRC, Thy1 and NST5 were measured relative to HPRT1 (median with an interquartile range of samples from three different donors represented; ${ }^{a} p<0.05$; Wilcoxon matched-pairs signed rank test). 
haematopoietic-lineage cells. Interestingly, when paired comparisons were conducted, CS was found to outperform BG with regards to MSC long-term retaining/proliferation.

\section{Effects of collagen membranes on BM-MSC osteogenesis}

Membranes were initially assessed for their support to osteogenesis when colonised by native BMMSCs. Thus, CS and BG were similarly loaded with BM aspirates (three different donors) for $3 \mathrm{~h}$, then cultured for 2 weeks in osteogenic or control expansion medium. Next, the gene expression levels of the osteogenic marker ALPL were measured. Interestingly, a marked, although not significant, increase in relative expression levels of ALPL was observed in BM cells cultured in osteogenic medium as compared to those cultured in expansion medium for both BG and CS (25- and 24-fold, respectively), with $A L P L$ transcript levels being higher in CS than BG (Fig. 5a).

Membranes were loaded with BM aspirates (two different donors) for $3 \mathrm{~h}$ and cultured for 3 weeks in osteogenic or control expansion medium. Then, the membranes were fixed and paraffin-wax-embedded. After cutting, sections were stained with alizarin red $S$ dye to visualise calcium deposition. Both membranes showed red-stained nodules when cultured in osteogenic medium as compared to expansion medium (Fig. 5b). These data indicated successful calcium deposition in osteogenic medium and that both BG and CS supported the osteogenic differentiation of native MSCs when membranes were loaded with BM aspirates.

The next step was to compare quantitatively the osteogenesis support offered by both BG and CS. However, this was limited by the low numbers of native MSCs in BM aspirates. Therefore, cultureexpanded BM-MSCs were used to load the collagen membranes for $3 \mathrm{~h}$ before culture in osteogenic medium for 2 weeks to study the activity of ALP by MSCs loaded on membranes. Consistently for all samples from six different donors, both collagen membranes significantly supported the cultureexpanded BM-MSC-derived ALP activity in the osteogenic condition as compared to the expansion condition ( $p=0.0312$ ) (Fig. 6a). Paired comparison of the ALP activity normalised to the DNA content between the two membranes demonstrated that CS supported ALP activity significantly more than BG in osteogenic conditions (2.5-fold, $p=0.0312$ ) (Fig. 6b).

To quantify calcium deposition, membranes were loaded for $3 \mathrm{~h}$ with culture-expanded BMMSCs and cultured for 3 weeks in osteogenic and a
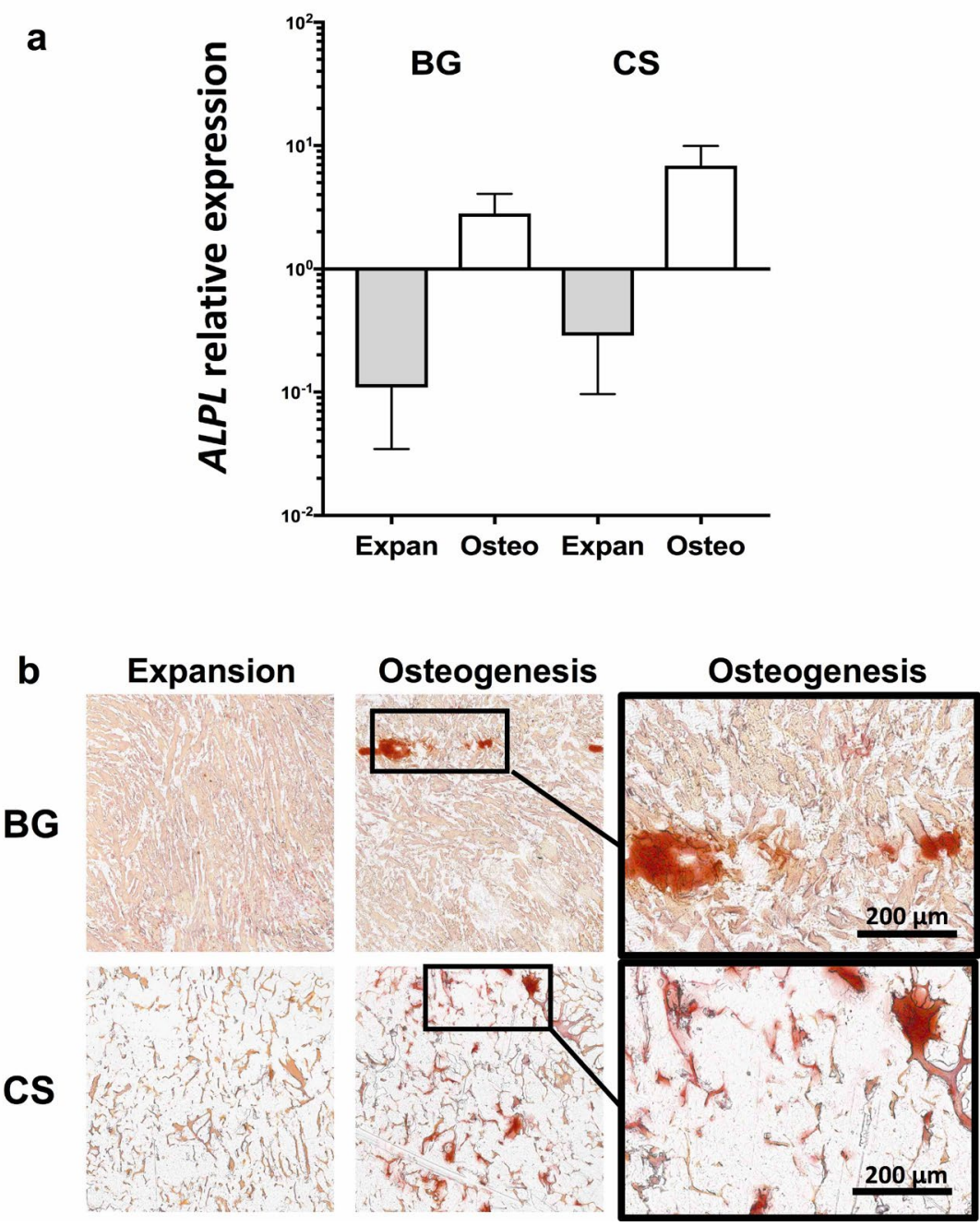

Fig. 5. MSC osteogenic differentiation for BM-aspirateloaded collagen membranes. (a) ALPL expression levels were measured relative to HPRT1. BG and CS were initially loaded for $3 \mathrm{~h}$ with $400 \mu \mathrm{L}$ of $\mathrm{BM}$ aspirate per $60 \mathrm{~mm}^{2}$ of each membrane. Then, the loaded membranes were cultured in expansion (Expan) or osteogenic (Osteo) medium for 1 or 3 weeks (median with an interquartile range of samples from three different donors represented). (b) Alizarin red S staining of the collagen membranes. BG and CS were initially loaded for $3 \mathrm{~h}$ with $\mathrm{BM}$ aspirate, then, they were cultured in expansion or osteogenic medium for 3 weeks. Arrows show the areas of alizarin red $S$ staining. 
a

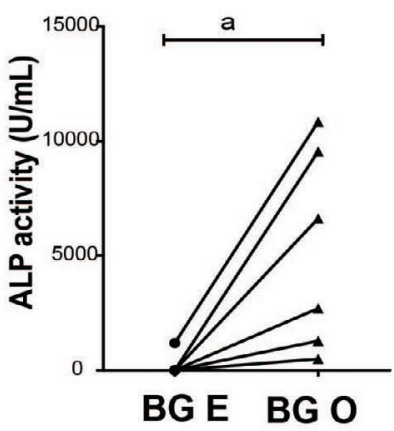

C

BG O
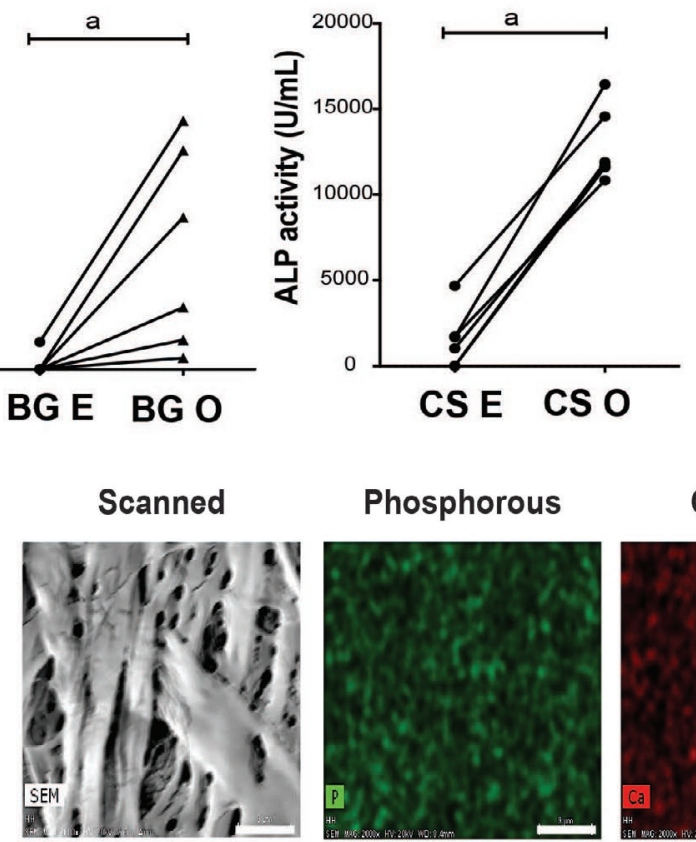
Phosphorous

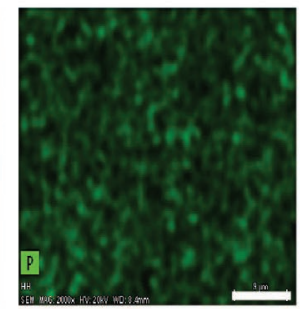

b

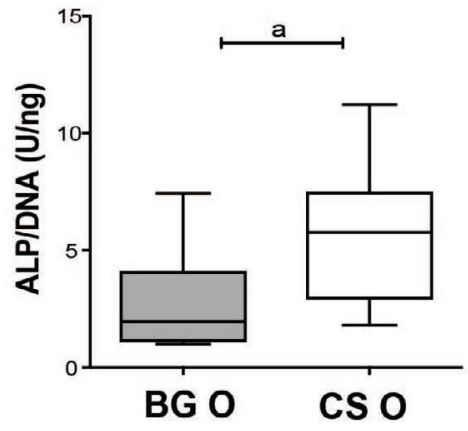

Calcium

Composite
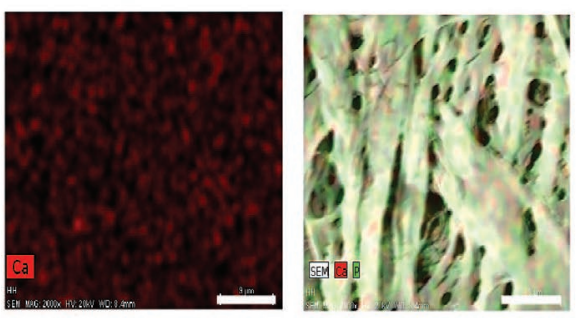

CS O
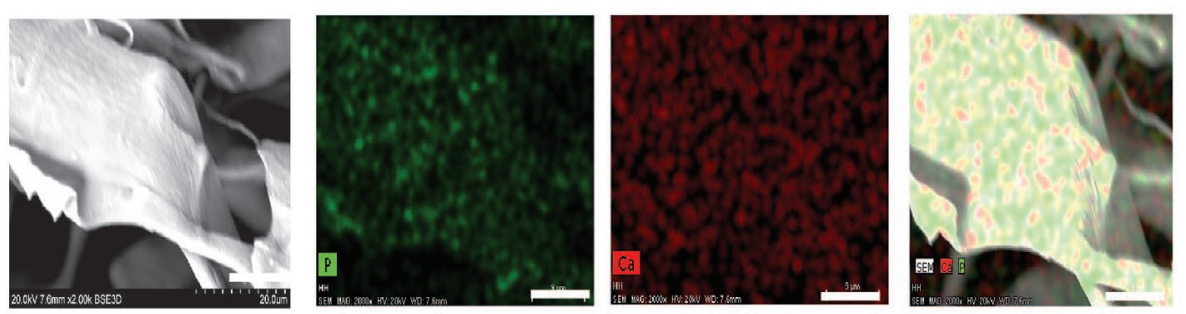

d

e
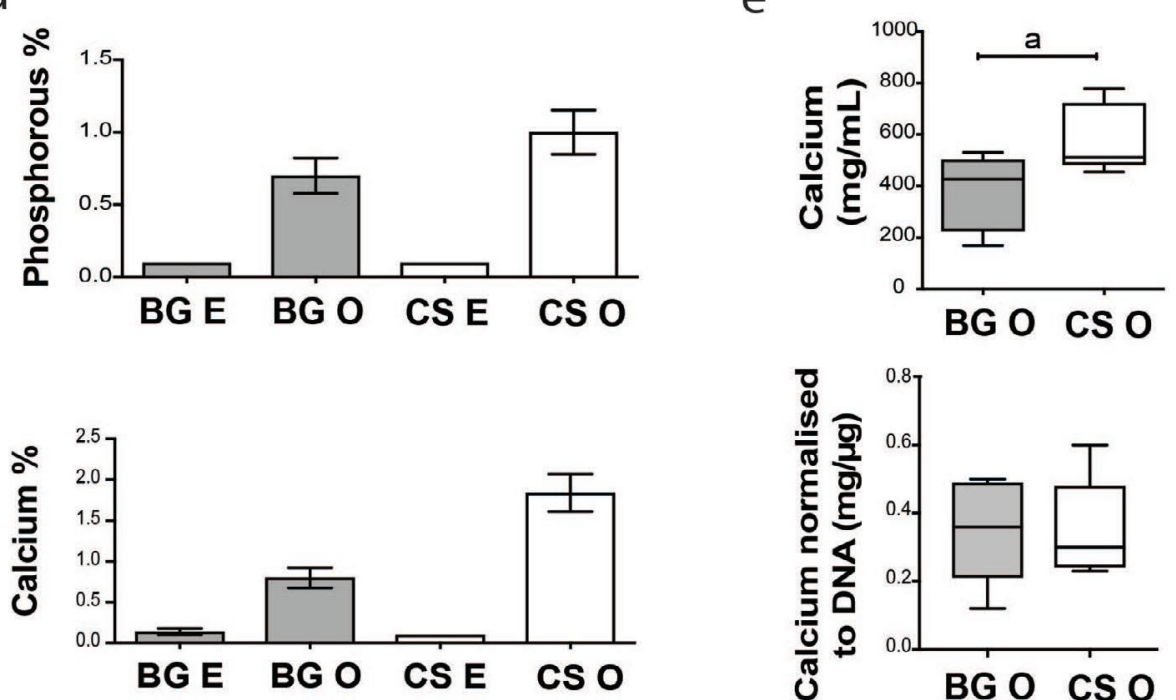

Fig. 6. Osteogenic differentiation of culture-expanded BM-MSCs loaded on collagen membranes. (a) ALP activity levels of culture-expanded BM-MSCs loaded on CS and BG and cultured in expansion (E) versus osteogenic (O) medium (6 different samples; ${ }^{a} p<0.05$, Wilcoxon matched-pairs signed rank test). (b) ALP activity normalised to DNA content (median of 6 samples with an interquartile range represented; ${ }^{\mathrm{a}} p<0.05$; Wilcoxon matched-pairs signed rank test). (c) EDS images for BG and CS loaded with culture-expanded BM-MSCs and cultured in osteogenic (O) medium. Scale bar: $9 \mu \mathrm{m}$. (d) Percentage of phosphorus (top) and calcium (bottom) among other elements detected using EDS for BG and CS. Mean of three samples with standard error is shown. (e) Total calcium levels for loaded cells as $\mathrm{mg} / \mathrm{mL}$ of $\mathrm{HCl}$ (the same volume was used for extraction in all samples) (upper panel), or calcium normalised to DNA as mg of calcium/ $\mu \mathrm{g}$ of DNA (lower panel) are shown. Bars show median with an interquartile range of samples from 5 different donors; ${ }^{a} p<0.05$, paired $t$-test. 
control expansion medium. A map of phosphorous and calcium deposition on the surface of BG and CS was obtained by EDS (Fig. 6c), confirming osteogenesis support by both collagen membranes. The percentages of phosphorous and calcium deposition by culture-expanded MSCs, relative to the sum of other detected elements (carbon, nitrogen and oxygen), were also quantified. A marked increase in the percentage of calcium and phosphorous was noted after 3 weeks of osteogenic culture relative to expansion cultures for both BG and CS (Fig. 6d). Also, colorimetric calcium assays were used to quantify calcium deposition levels and to confirm the imaging data. Remarkably, calcium levels extracted from CS loaded with culture-expanded BM-MSC were higher than that of BG (mean: 575 and $381 \mathrm{mg} / \mathrm{mL}, p=0.0073$ ) (Fig. 6e, upper panel). However, the calcium levels normalised to DNA content were similar for both CS and BG (Fig. 6e, lower panel). Altogether, these results showed that both collagen membranes supported equally the calcium deposition by BMMSCs, but higher ALP activity levels were noted for CS.

\section{Effect of collagen membranes on VEGF expression by BM-MSCs}

Collagen membranes were examined for their support to the angiogenic functions of native MSCs. To address this function, collagen membranes were loaded with BM aspirates for $3 \mathrm{~h}$ and cultured in expansion medium for 1 or 3 weeks. Gene expression levels of VEGF-A, PTPRS, THY1 and NST5 were measured by qPCR. Comparing 3-week to 1-week culture, while PTPRC levels decreased and Thy 1 and NST5 increased, a trend towards increased levels of $V E G F-A$ in BM cells after 3 weeks as compared to 1 week of culture was noted for both CS and BG (Fig. 7a). These data suggested that both collagen membranes supported the expression of the angiogenic factor VEGF-A in a mixture of BM cells, together with increased expression of MSC markers.

To confirm the observations noted for BM aspirates and to use enough cells for the quantitative comparison of VEGF-A between BG and CS, cultureexpanded MSCs were used. The two collagen membranes were loaded with culture-expanded BMMSCs for $3 \mathrm{~h}$ before culturing them in expansion or osteogenic medium. The levels of secreted VEGF-A were measured in the culture supernatants after 1 and 3 weeks by ELISA and normalised to cell number (samples from 5 different donors). Data demonstrated a consistent increase in normalised VEGF-A levels after 3 weeks of culture as compared to 1 week for both BG and CS in expansion (4.2- and 6.8-fold, $p=0.0102$ and $p=0.0040$, respectively) (Fig. $7 \mathbf{b}$ ) and osteogenic medium (4.9- and 3-folds, $p=0.0036$ and $p=0.0423$, respectively) (Fig. 7c). Comparing both culture conditions, the secreted VEGF-A levels (normalised to cell number) were lower in osteogenic cultures than in expansion cultures for BG and CS (Fig. 7b,c). Interestingly, paired comparisons for the membranes showed significantly higher levels of secreted normalised VEGF-A in the supernatants of CS as compared to BG after 3 weeks of culture in expansion medium ( $p=0.0302$ ) (Fig. 7b). However, no difference was noted between both membranes in osteogenic medium (Fig. 7c). In conclusion, these findings confirmed that both types of collagen membranes supported VEGF-A production by BMMSCs and that CS could support the secretion of VEGF-A, particularly from non-differentiating MSCs, better than BG.

To validate the secreted VEGF-A results at the cellular level, the gene expression levels for VEGF-A and $V E G F-C$ (the latter used to evaluate the specificity of VEGF-A expression levels) were measured relative to the housekeeping gene HPRT1. Three samples of culture-expanded BM-MSCs were loaded for $3 \mathrm{~h}$ on BG and CS and cultured for 1 and 3 weeks in expansion or osteogenic medium. Relative VEGF-C expression levels were consistently similar for BG and CS in both expansion and osteogenic conditions as well as between 1-week and 3-week cultures (Fig. 7d). In agreement with ELISA data, VEGF-A expression levels were significantly higher ( $>2$-fold difference) after 3 weeks than 1 week in osteogenic and expansion medium for both BG and CS ( $p=0.0312$; Fig. 7e). However, VEGF-A expression levels were consistently induced in osteogenic medium for both membranes as compared to expansion medium at week 3 (6-fold for BG and 4-fold for CS, $p=0.0312$ ) (Fig. 7e). Comparing cells loaded on BG and CS, $V E G F-A$ expression levels were higher for CS than BG when measured at 3 weeks (7-fold in expansion medium and 5-fold in osteogenic medium, both $p=0.0312$ ) (Fig. 7e). In summary, gene expression data showed that $V E G F-A$, but not $V E G F-C$, expression was increased over time in cultures of MSCloaded membranes and induced particularly by the osteogenic medium. Additionally, expression levels of secreted VEGF-A and its transcripts were consistently higher in BM-MSCs loaded on CS than on BG.

\section{Discussion}

The ideal membrane intended for augmenting GBR therapies must be mechanically flexible and biocompatible to enable maximum attachment and functions of MSCs (Jones et al., 2016; Polo-Corrales et al., 2014; Yousefi et al., 2016). BG is clinically used for GBR surgeries of dental applications to prevent ingrowth of soft tissue into the healing site and promote the appropriate bone and blood vessel development (Jung et al., 2013). BG is a noncrosslinked bilayer membrane formed of a one cell-non-permeable layer of tightly aligned collagen fibres, as shown by ESEM imaging (Fig. 1), to limit cell attachment and nutrient exchange. In comparison, the cell-permeable layer of BG allows cell ingrowth and nutrient/waste exchange. The second membrane 
a

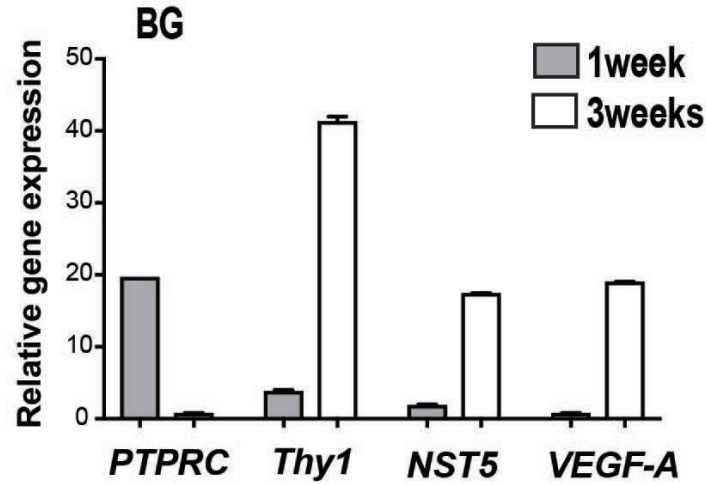

b

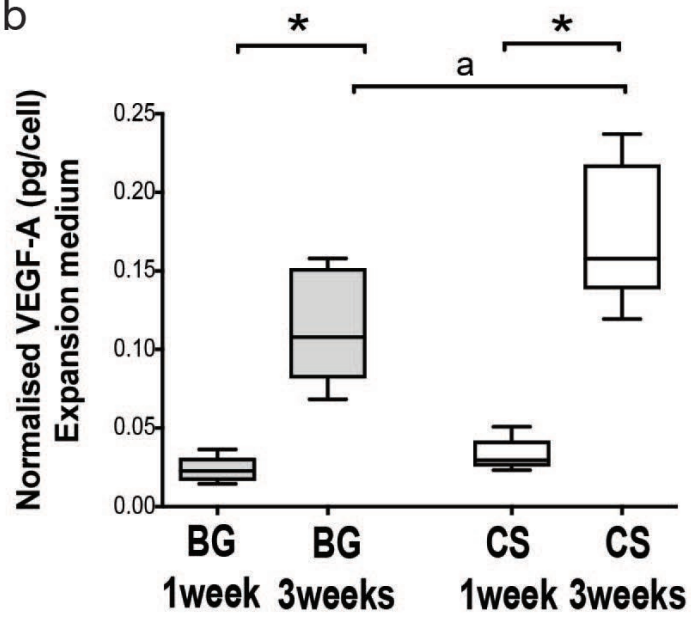

d

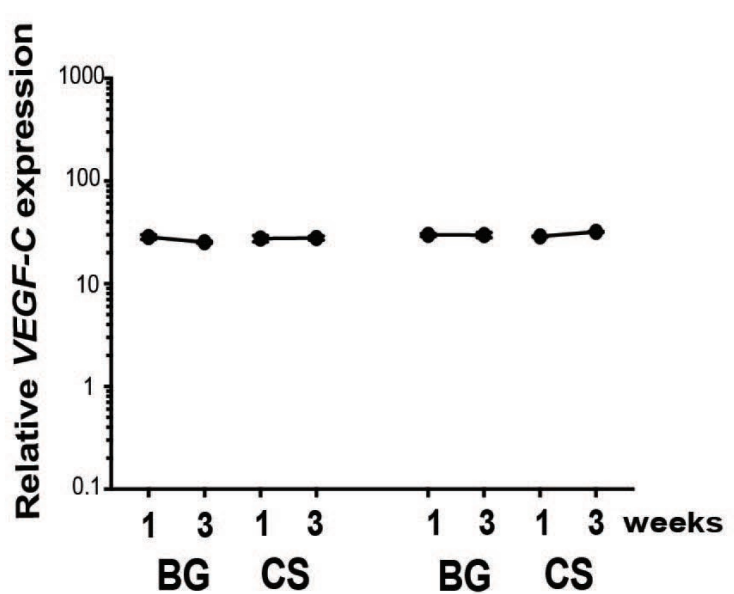

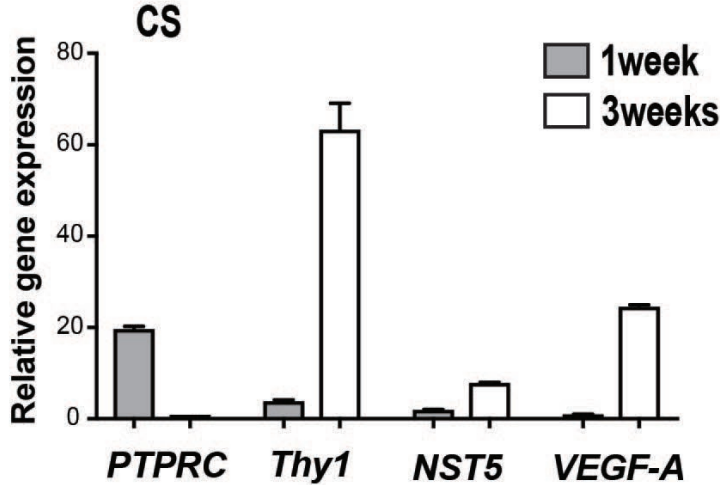

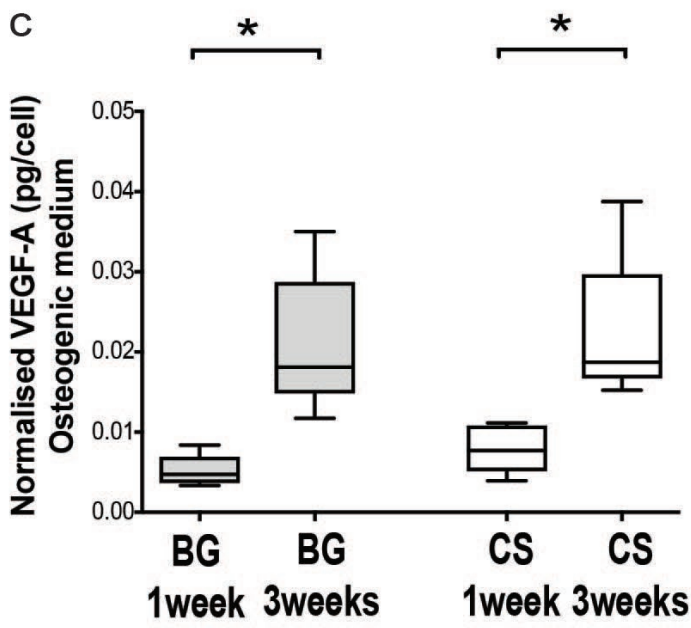

e

EXPANSION

OSTEOGENIC

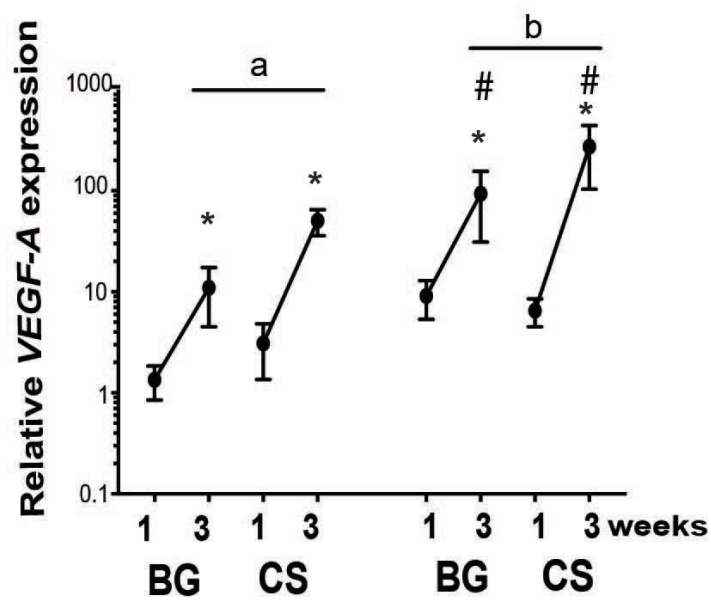

Fig. 7. VEGF expression by BM-MSCs loaded on collagen membranes. (a) Gene expression levels of cell lineage markers and VEGF-A were measured relative to HPRT1 (median with an interquartile range of 3 readings for one $\mathrm{BM}$ aspirate sample). $(\mathbf{b}, \mathbf{c})$ Normalised levels per cell numbers of secreted VEGF-A in expansion or osteogenic culture supernatants of membranes loaded with culture-expanded BM-MSCs. Median with an interquartile range of samples from 5 different donors represented. ${ }^{\mathrm{a}, \mathrm{b}, \mathrm{c}} p<0.05$, one-way ANOVA with Tukey's multiple comparisons test and Friedman test with Dunn's multiple comparison test were used for comparisons in $\mathbf{b}$ and $\mathbf{c}$, respectively. Gene expression levels of (d) VEGF-C and (e) $V E G F-A$ relative to HPRT1 were measured in membrane-loaded BM-MSCs. Median with an interquartile range of samples from 3 different donors represented, Wilcoxon matched-pairs signed rank test. ${ }^{a, b}$ indicate comparison between BG and CS; * between 1 week and 3 weeks; " between osteogenic and expansion; all indicate $p<0.05$. 
analysed in the study was CS, a thicker membrane formed of crosslinked collagen. How BG and CS membranes supported the attachment and osteogenic functions of human BM-MSCs was comprehensively tested. In addition, the attachment and survival of native MSCs from BM aspirates on these collagen membranes was examined. Both collagen membranes loaded with BM aspirates positively supported ALPL expression and calcium deposition in native MSCs. However, because of their rarity in BM aspirates, it was not feasible to quantify differences between both membranes regarding osteogenic differentiation and measuring VEGF-A expression. Therefore, these assays were performed using larger numbers of pure culture-expanded BM-MSCs.

Several dentistry-related studies have investigated the value of collagen-containing membranes for GBR, both in vitro and in vivo. For example, a nanoelectrospun bilayer collagen/chitosan membrane was shown to increase the osteogenic capacity of rabbit MSCs as assessed for calvarial defects (Lotfi et al., 2016). Also, natural collagenous nanofibrous membranes promote attachment, growth and osteogenic differentiation of rat or dog bonederived MSCs (Li et al., 2011; Yang et al., 2015). In contrast, the present study used human BM aspirate samples containing rare MSC populations as well as culture-expanded human BM-MSCs and tested their functional behaviour using various molecular and functional assays. Furthermore, the study demonstrated how small structural differences between the same material (i.e. collagen) could significantly affect attachment and, consequently, osteogenic functions of BM-MSCs. These data would contribute to a better understanding of the potential use of these membranes in the orthopaedic field.

ESEM and confocal microscopy were used to assess the topography of the membrane surface and to visualise MSC attachment (Fig. 1,2). However, it was technically challenging to determine native MSC attachment by microscopy. Therefore, flow cytometry was used to confirm the attachment of MSCs in their native status (in BM aspirates). The flowcytometry-based method applied was adapted from a previously published BM-MSC phenotype, identified as CD45 ${ }^{\text {negative }} \mathrm{CD} 271^{\text {high }}$, and using counting beads (El-Jawhari et al., 2017) to quantify the attachment of native BM-MSCs on the collagen membranes. Many studies have shown that using flow cytometry following collagenase digestion helps characterising and counting cells that colonise scaffolds or naturally exist in human bones (Baboolal et al. 2014; El-Jawhari et al., 2015; El-Jawhari et al., 2017; El-Sherbiny et al., 2018; Fragakis et al. 2018; Mirabella, et al. 2011). Both membranes successfully supported attachment and survival of BM-MSCs (Fig. 3,4). This finding agreed with what has been shown previously: i) the use of collagen-coated surfaces increases the adhesion of cultured human BM-MSCs (Somaiah et al., 2015); ii) collagen-containing scaffolds provide better attachment for these MSCs (El-Jawhari et al., 2015). The study data also indicated that other BM cells, including lymphocyte and myeloid-monocytelineage cells, were attached to BG or CS. However, similar percentages of BM cells were detected in pre-loading and post-loading samples, indicating no specific preferences for cell attachment during the early hours of incubation with the membranes. In addition, the number of haematopoietic cells was decreased over time for both BG and CS. In order to confirm flow cytometry data, particularly with regard to the low frequency and donor-related variability of BM-MSC number, real-time PCR was performed, assessing the time-dependent growth of different BM cell populations on the membranes. Gene expression data for cell lineage markers were consistent with the flow cytometry observations. Using both flow cytometry and real-time PCR, the preferential retention of MSCs $\left(\mathrm{CD} 45^{-} \mathrm{CD}^{+} 3^{+} \mathrm{CD} 90^{+}\right.$ cells), with a decline of haematopoietic-lineage cells (CD45 $5^{+}$population), on these collagen membranes was confirmed. These results were expected as the medium used (StemMACS ${ }^{\mathrm{TM}}$ MSC Expansion medium) is designed to support MSC expansion. Thus, haematopoietic-lineage cells were not or lessattached to the membranes' surfaces, resulting in their reduced survival. Additionally, the short half-life of the haematopoietic-lineage cells could be another factor causing reduced survival rates for these cell populations.

Interestingly, and despite donor-related variability of BM-MSC numbers, CS provided more support to BM-MSC attachment than BG, possibly because of the differences in their material properties (i.e. thickness and crosslinking of collagen). Collagen crosslinking could be a contributing factor for the superior performance of CS. In agreement with this, Rowland et al. (2013) indicated that collagen crosslinking of porcine cartilage-derived scaffold can positively affect human MSC adhesion. Other advantages of crosslinking are preventing collagen contraction caused by cell proliferation and reducing scaffold degradation rate (Lynn et al., 2004; Mafi et al., 2012; Rowland et al., 2013; Shoulders and Raines, 2009).

Both tested collagen membranes supported the osteogenesis of native BM-MSCs, as shown by the significant increase in $A L P L$ expression levels and calcium deposition when the loaded membranes were cultured in osteogenic medium (Fig. 5,6). These data agreed with previous human and animal studies showing that using ECM proteins, such as collagen and fibronectin, for scaffold fabrication enhances the osteogenic potential of BM and adipose-derived MSCs (Bernhardt et al., 2008; Chiu et al., 2012; Kang et al., 2013; Linsley et al., 2013; Serpooshan et al., 2010). Culture-expanded BM-MSCS were also used to load the collagen membranes, followed by ALP activity assays and calcium colorimetric assays, thus confirming previous data by using a 
sufficient number of native MSCs. In addition, a further increase in the duration of the osteogenic experiments (by at least 3 weeks) could have caused membrane degradation. Distinctively, the structure of the collagen scaffolds could affect ALP activity, with CS being superior. This finding could be explained by the larger area of MSC-collagen interactions within CS (because of higher thickness as compared to BG). Human BM-MSCs attached to the collagen matrix display augmented mineralisation concurrent with changes in their morphology and increased spreading area (Chiu et al., 2012). Collagen crosslinking of CS could be another explanation since the crosslinking of collagen gel enhances rat MSC osteogenic differentiation (Takitoh et al., 2015). While total calcium deposition was larger on CS than BG, calcium deposition normalised to BM-MSC count was similar for both CS and BG, confirming the effect of cell attachment. Collectively, the differences in osteogenesis between CS and BG are likely related to the structural differences, i.e. thickness difference and, accordingly, in the number of attached/survived MSCs.

VEGF-A plays a pivotal role during bone healing, with a paracrine influence on endothelial cells by boosting angiogenesis (Carlevaro et al., 2000; Hoch et al., 2012; Mayer et al., 2005; Street et al., 2002; Uchida et al., 2003). Immobilisation of VEGF-A or the use of a recombinant VEGF-A in collagen scaffolds augment angiogenesis and bone repair in animal models (Gao et al., 2013; Kim et al., 2016; Miyagi et al., 2011; Shen et al., 2008). The present study revealed that collagen membranes supported VEGF-A expression by human BM-MSCs in both expansion and differentiation medium (Fig. 7). These results partially agreed with the Mayer et al. (2005) study, which showed that VEGF-A secretion from human plastic-adherent BMMSCs is increased over time within the osteogenic medium. Comparing CS and BG, the secreted levels of VEGF-A in expansion cultures, as well as the gene expression levels of VEGF-A in osteogenic or expansion cultures, were higher in BM-MSCs attached to $\mathrm{CS}$. These finding can be related to the differences in attached cell numbers; as CS was thicker than BG and, accordingly, CS allowed more cell attachment. Interestingly, the study indicated VEGF-A induction in differentiating MSCs; however, the measured levels of secreted VEGF-A were inversely lower in osteogenic cultures than expansion cultures. The disparity regarding the expression levels of gene and secreted VEGF-A between differentiating and non-differentiating MSCs could be explained by the various functions of VEGF-A. Secreted VEGF-A helps in neo-vascularisation of bone tissue that precedes MSC differentiation into osteoblasts and deposition of minerals (Sivaraj et al., 2016). In contrast, intracellular VEGF-A seems to have an autocrine effect on differentiating MSCs regulating the expression of transcription factors favouring osteogenic differentiation, as shown in an animal study by Liu et al. (2012). The complex regulatory mechanisms of VEGF expression by MSCs suggests the importance of MSC-mediated angiogenesis and highlights the need for further investigations of MSC and endothelial cell interactions. While the tested collagen membranes supported the secretion of VEGF-A by colonised BM-MSCs, other MSC-related factors supporting new vessel formation have been reported, such as basic fibroblast growth factor (bFGF), transforming growth factor beta (TGF- $\beta$ ), platelet-derived growth factor (PDGF), angiopoietin-1, placental growth factor (PGF), interleukin-6 (IL-6) and certain miRNAs (Gong et al., 2017; Tao et al., 2016). These other angiogenic factors are also worth investigation to reveal how collagen membranes loaded with BM aspirate can support MSCs in their angiogenic functions.

In comparison to the two-stage induced membrane technique, using collagen membranes for augmenting antibiotic delivery would help avoiding the necessity for surgical insertion and removal of the cement spacer. Loading an absorbable type I bovine collagen membrane with antibiotics could reduce bacterial growth significantly (Cheng et al. 2015). However, further studies are also needed to assess in detail how antibiotic-loaded membranes can support MSC functions. Also, investigation of the functional effect of secreted VEGF-A on new vessel formation, e.g. by co-culture with endothelial cells, would be of value for future studies.

\section{Conclusions}

The present in vitro study demonstrated that both the crosslinked collagen membrane, CS, and the bilayer non-crosslinked membrane, BG, supported human BM-MSC attachment, proliferation, osteogenic differentiation and VEGF-A production. Differences in membrane thickness and, accordingly, cell attachment seemed to be the reason for the overall superior performance of CS. When loaded with $\mathrm{BM}$ aspirates containing native BM-MSCs, a membrane such as CS could be an attractive option in orthopaedics, promoting osteogenesis and angiogenesis in large bone defects. In contrast, a thinner bilayer collagen membrane such as BG could be potentially used as a barrier membrane with dual functions: the isolation of the healing area and the support of osteogenesis/angiogenesis. While the study highlighted the advantages of these collagen membranes, future studies using other materials would help understanding the collagen-specific effect versus other natural or synthetic materials for GBR orthopaedic therapies. Additionally, performing in vivo work would be needed to confirm the functional impact of inducting MSC angiogenic and osteogenic activities when these cells colonise collagen membranes in vivo. 


\section{Acknowledgements}

This work was supported by an educational grant from Geistlich Pharma, Wolhusen, Switzerland [grant number: 101930]. The funder did not contribute to project design, sample collection, data analysis or results. The authors gratefully acknowledge the help given by the orthopaedic theatre team in Leeds General Infirmary and Heather Owston and Payal Ganguly for their help with sample collection. Also, the authors thank Jackie Hudson for her help with ESM and EDS. JJE is part-funded by AO Foundation start-up grant (grant number S-16-132E). KM is funded by the EPSRC Centre for Doctoral Training in Tissue Engineering and Regenerative Medicine Innovation in Medical and Biological Engineering multidisciplinary collaboration of Faculties at the University of Leeds (grant number EP/L014823/1).

\section{References}

Alpar B, Leyhausen G, Gunay H, Geurtsen W (2000) Compatibility of resorbable and nonresorbable guided tissue regeneration membranes in cultures of primary human periodontal ligament fibroblasts and human osteoblast-like cells. Clin Oral Investig 4: 219-225.

Anagnostakos K (2017) Therapeutic use of antibiotic-loaded bone cement in the treatment of hip and knee joint infections. J Bone Jt Infect 2: 29-37.

Arrington ED, Smith WJ, Chambers HG, Bucknell AL, Davino NA (1996) Complications of iliac crest bone graft harvesting. Clin Orthop Relat Res: 300-309.

Baboolal TG, Boxall SA, El-Sherbiny YM, Moseley TA, Cuthbert RJ, Giannoudis PV, McGonagle D, Jones E (2014) Multipotential stromal cell abundance in cellular bone allograft: comparison with fresh agematched iliac crest bone and bone marrow aspirate. Regen Med 9: 593-607.

Battula VL, Treml S, Bareiss PM, Gieseke F, Roelofs H, de Zwart P, Muller I, Schewe B, Skutella T, Fibbe WE, Kanz L, Buhring HJ (2009) Isolation of functionally distinct mesenchymal stem cell subsets using antibodies against CD56, CD271, and mesenchymal stem cell antigen-1. Haematologica 94: 173-184.

Bernhardt A, Lode A, Boxberger S, Pompe W, Gelinsky M (2008) Mineralised collagen - an artificial, extracellular bone matrix-improves osteogenic differentiation of bone marrow stromal cells. J Mater Sci Mater Med 19: 269-275.

Carlevaro MF, Cermelli S, Cancedda R, Descalzi Cancedda F (2000) Vascular endothelial growth factor (VEGF) in cartilage neovascularization and chondrocyte differentiation: auto-paracrine role during endochondral bone formation. J Cell Sci 113: 59-69.

Cheng CF, Wu KM, Chen YT, Hung SL (2015) Bacterial adhesion to antibiotic-loaded guided tissue regeneration membranes -a scanning electron microscopy study. J Formos Med Assoc 114: 35-45.

Chevallay B, Herbage D (2000) Collagenbased biomaterials as 3D scaffold for cell cultures: applications for tissue engineering and gene therapy. Med Biol Eng Comput 38: 211-218.

Chiu LH, Yeh TS, Huang HM, Leu SJ, Yang CB, Tsai YH (2012) Diverse effects of type II collagen on osteogenic and adipogenic differentiation of mesenchymal stem cells. J Cell Physiol 227: 2412-2420.

Churchman SM, Ponchel F, Boxall SA, Cuthbert R, Kouroupis D, Roshdy T, Giannoudis PV, Emery P, McGonagle D, Jones EA (2012) Transcriptional profile of native CD271+ multipotential stromal cells: evidence for multiple fates, with prominent osteogenic and Wnt pathway signaling activity. Arthritis and rheumatism 64: 2632-2643.

Crisostomo PR, Wang Y, Markel TA, Wang M, Lahm T, Meldrum DR (2008) Human mesenchymal stem cells stimulated by TNF-alpha, LPS, or hypoxia produce growth factors by an NF kappa B- but not JNK-dependent mechanism. Am J Physiol Cell Physiol 294: C675-682.

Cuthbert R, Boxall SA, Tan HB, Giannoudis PV, McGonagle D, Jones E (2012) Single-platform quality control assay to quantify multipotential stromal cells in bone marrow aspirates prior to bulk manufacture or direct therapeutic use. Cytotherapy 14: 431-440.

Cuthbert RJ, Churchman SM, Tan HB, McGonagle D, Jones E, Giannoudis PV (2013) Induced periosteum a complex cellular scaffold for the treatment of large bone defects. Bone 57: 484-492.

Dimitriou R, Mataliotakis GI, Calori GM, Giannoudis PV (2012) The role of barrier membranes for guided bone regeneration and restoration of large bone defects: current experimental and clinical evidence. BMC Med 10: 81. DOI: 10.1186/1741-701510-81.

Dominici M, Le Blanc K, Mueller I, SlaperCortenbach I, Marini F, Krause D, Deans R, Keating A, Prockop D, Horwitz E (2006) Minimal criteria for defining multipotent mesenchymal stromal cells. The International Society for Cellular Therapy position statement. Cytotherapy 8: 315-317.

Elgali I, Omar O, Dahlin C, Thomsen P (2017) Guided bone regeneration: materials and biological mechanisms revisited. Eur J Oral Sci 125: 315-337.

El-Jawhari JJ, Cuthbert R, McGonagle D, Jones E, Giannoudis PV (2017) The CD45lowCD271high cell prevalence in bone marrow samples may provide a useful measurement of the bone marrow quality for cartilage and bone regenerative therapy. J Bone Joint Surg Am 99: 1305-1313.

El-Jawhari JJ, Sanjurjo-Rodriguez C, Jones E, Giannoudis PV (2015) Collagen-containing scaffolds enhance attachment and proliferation of non-cultured bone marrow multipotential stromal cells. J Orthop Res 34: 597-606.

El-Sherbiny YM, El-Jawhari JJ, Moseley TA, McGonagle D and Jones E (2018) T cell immunomodulation by clinically used allogeneic 
human cancellous bone fragments: a potential novel immunotherapy tool. Sci Rep 8: 13535. DOI: 10.1038/ s41598-018-31979-1.

Fragkakis EM, El-Jawhari JJ, Dunsmuir RA, Millner PA, Rao AS, Henshaw KT, Pountos I, Jones E, Giannoudis PV (2018) Vertebral body versus iliac crest bone marrow as a source of multipotential stromal cells: comparison of processing techniques, tri-lineage differentiation and application on a scaffold for spine fusion. PLoS One 13: e0197969. DOI: 10.1371/journal. pone.0197969.

Gao C, Harvey EJ, Chua M, Chen BP, Jiang F, Liu Y, Li A, Wang H, Henderson JE (2013) MSC-seeded dense collagen scaffolds with a bolus dose of VEGF promote healing of large bone defects. Eur Cell Mater 26: 195-207.

Garg P, Ranjan R, Bandyopadhyay U, Chouksey S, Mitra S, Gupta SK (2011) Antibiotic-impregnated articulating cement spacer for infected total knee arthroplasty. Indian J Orthop 45: 535-540.

Giannoudis PV, Faour O, Goff T, Kanakaris N, Dimitriou R (2011) Masquelet technique for the treatment of bone defects: tips-tricks and future directions. Injury 42: 591-598.

Gong M, Yu B, Wang J, Wang Y, Liu M, Paul C, Millard RW, Xiao DS, Ashraf M and Xu M (2017) Mesenchymal stem cells release exosomes that transfer miRNAs to endothelial cells and promote angiogenesis. Oncotarget 8: 45200-45212.

Hammerle CH, Jung RE (2003) Bone augmentation by means of barrier membranes. Periodontol 2000 33: 36-53.

Hennessy KM, Pollot BE, Clem WC, Phipps MC, Sawyer AA, Culpepper BK, Bellis SL (2009) The effect of collagen I mimetic peptides on mesenchymal stem cell adhesion and differentiation, and on bone formation at hydroxyapatite surfaces. Biomaterials 30: 1898-1909.

Hoch AI, Binder BY, Genetos DC, Leach JK (2012) Differentiation-dependent secretion of proangiogenic factors by mesenchymal stem cells. PLoS One 7: e35579. DOI: 10.1371/journal.pone.0035579.

Jones EA, Giannoudis PV, Kouroupis D (2016) Bone repair with skeletal stem cells: rationale, progress to date and clinical application. Ther Adv Musculoskelet Dis 8: 57-71.

Jung RE, Fenner N, Hammerle CH, Zitzmann NU (2013) Long-term outcome of implants placed with guided bone regeneration (GBR) using resorbable and non-resorbable membranes after 12-14 years. Clin Oral Implants Res 24: 1065-1073.

Kang BJ, Kim Y, LeeSH, Kim WH, Woo HM, Kweon OK (2013) Collagen I gel promotes homogenous osteogenic differentiation of adipose tissue-derived mesenchymal stem cells in serum-derived albumin scaffold. J Biomater Sci Polym Ed 24: 1233-1243.

Kim JH, Kim TH, Kang MS, Kim HW (2016) Angiogenic effects of collagen/mesoporous nanoparticle composite scaffold delivering VEGF165. Biomed Res Int 2016: 9676934. DOI: $10.1155 / 2016 / 9676934$.
Kouroupis D, Baboolal TG, Jones E, Giannoudis PV (2013) Native multipotential stromal cell colonization and graft expander potential of a bovine natural bone scaffold. J Orthop Res 31: 1950-1958.

Lee SW, Kim SG (2014) Membranes for the guided bone regeneration. Maxillofac Plast Reconstr Surg 36: 239-246.

Li X, Chen SL, Huang DY, Zhu SX and Wang JL (2011) Maxillary sinus augmentation with simultaneous implantation using guided bone regeneration. Zhonghua Kou Qiang Yi Xue Za Zhi 46: 547-550.

Linsley C, Wu B, Tawil B (2013) The effect of fibrinogen, collagen type I, and fibronectin on mesenchymal stem cell growth and differentiation into osteoblasts. Tissue Eng Part A 19: 1416-1423.

Liu Y, Berendsen AD, Jia S, Lotinun S, Baron R, Ferrara N, Olsen BR (2012) Intracellular VEGF regulates the balance between osteoblast and adipocyte differentiation. J Clin Invest 122: 3101-3113.

Lotfi G, Shokrgozar MA, Mofid R, Abbas FM, Ghanavati F, Baghban AA, Yavari SK, Pajoumshariati $S$ (2016) Biological evaluation (in vitro and in vivo) of bilayer collagenous coated (nano electrospun and solid wall) chitosan membrane for periodontal guided bone regeneration. Ann Biomed Eng 44: 21322144.

Lynn AK, Yannas IV, Bonfield W (2004) Antigenicity and immunogenicity of collagen. J Biomed Mater Res B Appl Biomater 71: 343-354.

Mafi P, Hindocha S, Mafi R, Khan WS (2012) Evaluation of biological protein-based collagen scaffolds in cartilage and musculoskeletal tissue engineering a systematic review of the literature. Curr Stem Cell Res Ther 7: 302-309.

Marouf HA, El-Guindi HM (2000) Efficacy of highdensity versus semipermeable PTFE membranes in an elderly experimental model. Oral Surg Oral Med Oral Pathol Oral Radiol Endod 89: 164-170.

Masquelet AC, Begue T (2010) The concept of induced membrane for reconstruction of long bone defects. Orthop Clin North Am 41: 27-37.

Mayer H, Bertram H, Lindenmaier W, Korff T, Weber H, Weich H (2005) Vascular endothelial growth factor (VEGF-A) expression in human mesenchymal stem cells: autocrine and paracrine role on osteoblastic and endothelial differentiation. J Cell Biochem 95: 827-839.

Mirabella T, Poggi A, Scaranari M, Mogni M, Lituania M, Baldo C, Cancedda R, Gentili C (2011) Recruitment of host's progenitor cells to sites of human amniotic fluid stem cells implantation. Biomaterials 32: 4218-4227.

Miyagi Y, Chiu LL, Cimini M, Weisel RD, Radisic M, Li RK (2011) Biodegradable collagen patch with covalently immobilized VEGF for myocardial repair. Biomaterials 32: 1280-1290.

Polo-Corrales L, Latorre-Esteves M, Ramirez-Vick JE (2014) Scaffold design for bone regeneration. J Nanosci Nanotechnol 14: 15-56. 
Popov C, Radic T, Haasters F, Prall WC, Aszodi A, Gullberg D, Schieker M, Docheva D (2011) Integrins alpha2beta1 and alpha11beta1 regulate the survival of mesenchymal stem cells on collagen I. Cell Death Dis 2: e186. DOI: 10.1038/cddis.2011.71.

Rowland CR, Lennon DP, Caplan AI, Guilak F (2013) The effects of crosslinking of scaffolds engineered from cartilage ECM on the chondrogenic differentiation of MSCs. Biomaterials 34: 5802-5812.

Schwarz F, Schmucker A, Becker J (2017) Longterm outcomes of simultaneous guided bone regeneration using native and cross-linked collagen membranes after 8 years. Clin Oral Implants Res 28: 779-784.

Serpooshan V, Julien M, Nguyen O, Wang H, Li A, Muja N, Henderson JE, Nazhat SN (2010) Reduced hydraulic permeability of three-dimensional collagen scaffolds attenuates gel contraction and promotes the growth and differentiation of mesenchymal stem cells. Acta Biomater 6: 3978-3987.

Shen YH, Shoichet MS, Radisic M (2008) Vascular endothelial growth factor immobilized in collagen scaffold promotes penetration and proliferation of endothelial cells. Acta Biomater 4: 477-489.

Shoulders MD, Raines RT (2009) Collagen structure and stability. Annu Rev Biochem 78: 929958.

Sivakumar R, Mohideen MG, Chidambaram M, Vinoth T, Singhi PK, Somashekar V (2016) Management of large bone defects in diaphyseal fractures by induced membrane formation by Masquelet's technique. J Orthop Case Rep 6: 59-62.

Sivaraj KK, Adams RH (2016) Blood vessel formation and function in bone. Development 143: 2706-2715.

Somaiah C, Kumar A, Mawrie D, Sharma A, Patil SD, Bhattacharyya J, Swaminathan R, Jaganathan BG (2015) Collagen promotes higher adhesion, survival and proliferation of mesenchymal stem cells. PLoS One 10: e0145068. DOI: 10.1371/journal.pone.0145068.

Stoppel WL, Ghezzi CE, McNamara SL, Black LD, 3rd, Kaplan DL (2015) Clinical applications of naturally derived biopolymer-based scaffolds for regenerative medicine. Ann Biomed Eng 43: 657-680.

Street J, Bao M, deGuzman L, Bunting S, Peale FV, Jr., Ferrara N, Steinmetz H, Hoeffel J, Cleland JL, Daugherty A, van Bruggen N, Redmond HP, Carano RA, Filvaroff EH (2002) Vascular endothelial growth factor stimulates bone repair by promoting angiogenesis and bone turnover. Proc Natl Acad Sci U S A 99: 9656-9661.

Takitoh T, Bessho M, Hirose M, Ohgushi H, Mori H, Hara M (2015) Gamma-cross-linked nonfibrillar collagen gel as a scaffold for osteogenic differentiation of mesenchymal stem cells. J Biosci Bioeng 119: $217-$ 225.

Tao H, Han Z, Han ZC and Li Z (2016) Proangiogenic features of mesenchymal stem cells and their therapeutic applications. Stem Cells Int 2016: 1314709. DOI: 10.1155/2016/1314709.
Uchida S, Sakai A, Kudo H, Otomo H, Watanuki M, Tanaka M, Nagashima M, Nakamura T (2003) Vascular endothelial growth factor is expressed along with its receptors during the healing process of bone and bone marrow after drill-hole injury in rats. Bone 32: 491-501.

Yang M, Zhou G, Castano-Izquierdo H, Zhu $Y$ and Mao C (2015) Biomineralization of natural collagenous nanofibrous membranes and their potential use in bone tissue engineering. J Biomed Nanotechnol 11: 447-456.

Yousefi AM, James PF, Akbarzadeh R, Subramanian A, Flavin C, Oudadesse H (2016) Prospect of stem cells in bone tissue engineering a review. Stem Cells Int 2016: 6180487. DOI: 10.1155/2016/6180487.

\section{Discussion with Reviewer}

Marietta Herrmann: In this study, a one-step procedure for seeding fresh BM aspirate on collagen membranes was suggested as a GBR strategy. The authors demonstrated that different cell populations attached to the membranes, including haematopoietic cell populations. Could the authors speculate which contribution different haematopoietic cell populations might have in bone tissue regeneration? Authors: Several studies indicated that haematopoietic cell populations have an important role during all three phases of normal bone regeneration following fracture. During the early phase, myeloid-lineage cells, particularly macrophages and neutrophils, help clearing tissue debris and prepare MSCs for osteogenesis (cytokine-mediated priming process) (Krampera, 2011; Han et al., 2014; Grassi et al., 2016; Tso et al., 2010, additional reference). During the repair phase, macrophages, $\mathrm{T}$ - and B-lymphocytes and various cytokines and growth factors produced by these cells are involved in conversion of soft callus into hard callus and formation of new blood vessels (El-Jawhari et al., 2016; Gong et al., 2016; Könnecke et al., 2014; Spiller et al., 2014, additional references). During the bone remodelling phase, interactions between macrophage-derived osteoclasts, MSCderived osteoblasts and, probably, Th17 lymphocytes are critical to achieve bone union (Cho, 2015; Huang et al., 2009, additional references). With the use of bioabsorbable collagen membranes as GBR devices, the presence of haematopoietic cell populations within the membranes is probably most valuable at the early stages following implantation, i.e. to prime MSCs for osteogenesis.

The present study showed that the BM haematopoietic cell populations survived within the membranes for a limited time in in vitro culture conditions. In a future study, it would be interesting to test if the presence of these cells has a specific MSC priming effect. Overall, testing the co-cultures of specific types of immune cells with MSCs using different materials will be of great importance to 
further understand the role of haematopoietic cells in bone healing and to help optimise bone regenerative therapies.

\section{Additional References}

Cho SW (2015) Role of osteal macrophages in bone metabolism. J Pathol Transl Med 49: 102-104.

El-Jawhari JJ, Jones E, Giannoudis, PV (2016) The roles of immune cells in bone healing; what we know, do not know and future perspectives. Injury 47: 23992406.

Gong L, Zhao Y, Zhang Y, Ruan Z (2016) The macrophage polarization regulates MSC osteoblast differentiation in vitro. Ann Clin Lab Sci 46: 65-71.

Grassi F, Cattini L, Gambari L, Manferdini C, Piacentini A, Gabusi E, Facchini A, Lisignoli G (2016) T cell subsets differently regulate osteogenic differentiation of human mesenchymal stromal cells in vitro. J Tissue Eng Regen Med 10: 305-314.

Han X, Yang Q, Lin L, Xu C, Zheng C, Chen X, Han Y, Li M, Cao W, Cao K, Chen Q, Xu G, Zhang Y, Zhang J, Schneider RJ, Qian Y, Wang Y, Brewer G, Shi Y (2014) Interleukin-17 enhances immunosuppression by mesenchymal stem cells. Cell Death Differ 21: 1758-1768.
Huang H, Kim HJ, Chang EJ, Lee ZH, Hwang SJ, Kim HM, Lee Y, Kim HH (2009) IL-17 stimulates the proliferation and differentiation of human mesenchymal stem cells: implications for bone remodeling. Cell Death Differ 16: 1332-1343.

Könnecke I, Serra A, El Khassawna T, Schlundt C, Schell H, Hauser A, Ellinghaus A, Volk HD, Radbruch A, Duda GN, Schmidt-Bleek K (2014) T and B cells participate in bone repair by infiltrating the fracture callus in a two-wave fashion. Bone 64: 155-165.

Krampera M (2011) Mesenchymal stromal cell 'licensing': a multistep process. Leukemia 25: 14081414.

Spiller KL, Anfang RR, Spiller KJ, Ng J, Nakazawa KR, Daulton JW, Vunjak-Novakovic (2014) The role of macrophage phenotype in vascularization of tissue engineering scaffolds. Biomaterials 35: 4477-4488.

Tso GH, Law HK, Tu W, Chan GC, Lau YL (2010) Phagocytosis of apoptotic cells modulates mesenchymal stem cells osteogenic differentiation to enhance IL-17 and RANKL expression on CD4+ T cells. Stem Cells 28: 939-954.

Editor's note: The Scientific Editor responsible for this paper was Mauro Alini. 